\title{
Effect of Different Concentrations of Indole-3-butyric Acid and Different Cutting Size (Length and Diameter) on Shoot Growth of Pomegranate (Punica granatum L.) Cuttings
}

\author{
Basir Ahmad Ahmadi ${ }^{\text {** }}$, G. M. Waghmare ${ }^{2}$ and A. M. Bhosale ${ }^{1}$ \\ ${ }^{1}$ Department of Horticulture, VNMKV., Parbhani, India \\ ${ }^{2}$ Department of Agriculture, Badnapur, VNMKV., Parbhani, India \\ *Corresponding author
}

\section{Keywords}

Cuttings, Indole-3butyric acid, Shoot growth,

Pomegranate, FRBD

\section{Article Info}

\section{Accepted:}

10 January 2021

Available Online:

10 February 2021

\section{A B S T R A C T}

Result revealed that, significantly minimum days to sprout, highest sprouting percentage, maximum number of branches, maximum length and diameter of shoot, highest number of leaves per cutting, highest leaf area and finally shoot to root ratio of pomegranate cutting was recorded with treatment $\mathrm{D}_{2}$ i.e. (1.5 $\mathrm{cm}$ diameter) and maximum days to sprout and minimum growth parameters were observed in $\mathrm{D}_{1}$ i.e. (1 $\mathrm{cm}$ diameter) minimum days to sprout, highest sprouting percentage, maximum number of branches, maximum length and diameter of shoot, highest number of leaves per cutting, highest leaf area and finally shoot to root ratio of pomegranate cutting was recorded with treatment $\mathrm{L}_{3}$ i.e. (20 cm length) and lowest values were observed in $\mathrm{L}_{1}$ i.e. $(10 \mathrm{~cm}$ length) and minimum days to sprout, highest sprouting percentage, maximum number of branches, maximum length and diameter of shoot, highest number of leaves per cutting, highest leaf area and finally shoot to root ratio of pomegranate cutting were recorded with treatment $T_{1}$ i.e. (IBA $3000 \mathrm{ppm}$ ) and lowest growth parameters were found in treatment $\mathrm{T}_{2}$ i.e. (IBA $4000 \mathrm{ppm}$ ).

\section{Introduction}

Pomegranate (Punica granatum L.) belongs to family Punicaceae is native to Asia especially to Iran, Afghanistan and Himalayan region. It is one of the oldest known edible fruit of tropical and sub-tropical region, known for its gustative, medicinal and ornamental. The tree is quite resistant to cold when dormant, withstanding temperatures down to $10^{\circ} \mathrm{F}$. However, it is very sensitive to frost before it reaches full dormancy in late fall and after buds have begun to swell in early spring.

The best quality pomegranate fruits are produced in regions with cool winters and hot, dry summers. Pomegranate is a shrub that naturally tends to develop multiple trunks and 
has a bushy appearance. When domesticated, it is grown as a small tree that grows up to $5 \mathrm{~m}$, leaves have an oblanceolate shape with an apex and acuminate base. Mature leaves are green, entire, smooth and hairless; the flowers can appear solitary, pairs or cluster. In the most cases, the solitary flowers will appear as spurs along the branches while the clusters are terminal (Levin, 2006). Fruit is globular, possessing a smooth outer rind with juicy arils (Mars, 2000).

Pomegranate could be propagated either sexually by seeds or vegetatively using stem cuttings and sometimes as layers or suckers or by grafting (Hartmann et al., 1997). However, in commercial propagation stem cuttings are widely used (Antakya Hatay, 2009).

The length and diameter of stem cuttings have an impact on rooting rate and subsequent survival in the field after transplanting, determining optimal cutting length is essential as (1) a very long cutting larger than $12 \mathrm{~cm}$ will be waste of valuable coppice material, with limited or no benefit in rooting percentage, whereas, (2) a short cutting may not result in the development of sufficient roots (possibly due to lack of sufficient storage reserves) (Leakey, 2004).

Sprouting and rooting ability of cuttings is mainly depends upon the physiological maturity of the shoot and conditions where cuttings have been planted for sprouting and subsequent rooting (Purohit and Shekrappa, 1985) and various internal and external factors like seasons and concentration of endogenous and exogenous phytohormones (Arya et al., 1994).

Plant growth regulators improve the rooting of cutting by stimulating the production of adventitious roots. Went (1934) first postulated that auxins initiate adventitious root formation in stem cuttings.
IBA is the most promising growth regulator inducing rooting quickly. Exogenous application of IBA accelerates the rate of rooting, increases final rooting percentage and number of roots. However, relatively high concentrations of IBA have been reported to be inhibitory to rooting (Leakey, 1990). It promotes root initiation, number of roots and shoots growth in number of ornamental and fruits plants. The rooting medium can have a major influence on the rooting capacity of cuttings (Hartmann et al., 2002).

\section{Materials and Methods}

The experiment was carried out during the year 2017-18 at Department of Horticulture, Vasantrao Naik Marathwada Krishi Vidyapeeth, Parbhani on pomegranate (Punica granatum L.) cv. Bhagwa. The cuttings were taken from hardwood cuttings arising on trees of pomegranate $\mathrm{cv}$. Bhagwa during the month of July. Experiment was laid out in Factorial Randomized Block Designed (FRBD) with 18 treatments and 3 factors which are replicated trice and no. of cuttings per treatment are 40 .

\section{Treatment Details}

\section{Observation recorded}

\section{Shoot growth observation}

\section{Days to sprouting}

The planted cuttings were observed daily under each treatment and the number of days required for sprouting was recorded and mean was calculated as days taken for first sprout to appear.

\section{Sprouting percentage}

The per cent sprouting was counted at 30 days after planting by taking the ratio of number of cuttings sprouted to the number of cuttings planted and multiplied by 100 . 
It can be calculated by using following formula.

$$
\text { Percentage of sprouted cuttings }=\frac{\text { No. of cuttings sprouted }}{-} \text { Total no. of cutting }
$$

\section{Number of shoot per cutting}

On the $30^{\text {th }}, 60^{\text {th }}$ and $90^{\text {th }}$ day after planting, the number of sprouts per cutting was counted and their mean was used to record this parameter.

\section{Length of shoots per cutting}

The length of longest shoot per cutting was measured after 60 and 90 days after planting and its mean was expressed in centimeters.

Five cuttings in each replication were used for recording the length of longest shoot per cutting.

\section{Diameter of shoots per cutting}

The diameter of shoot per cutting was measured after 60 and 90 days after planting and its mean was expressed in millimeters. Five cuttings in each replication were used for recording the diameter of shoot per cutting.

\section{Shoot fresh and dry weight (g)}

All the shoot of each selected fifteen cuttings from three replications were removed with the help of secator.

The separate fresh shoots were placed in brown paper bags, properly labeled, and subsequent weighted.

After that for determination of dry shoot weight, shoots placed in oven at $60{ }^{\circ} \mathrm{C}$ and after stabilization of weight, this stabilized weight (g) was recorded. From the fresh weight of shoot, we can find out the dry weight of shoot by using the following formula.

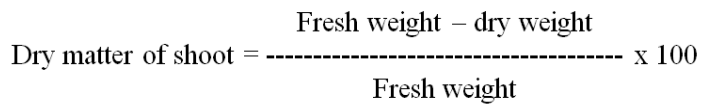

\section{Shoot to root ratio}

Shoot to root ratio dry and fresh basis are calculated using the following formula.

$$
\begin{gathered}
\text { Fresh basis }=\text { - } \begin{array}{c}
\text { Fresh weight of root } \\
\text { Fresh weight of shoot } \\
\text { Dry basis }=
\end{array} \text { - Dry weight of root } \\
\text { Dry weight of shoot }
\end{gathered}
$$

\section{Number of leaves per cutting}

On the 30, 60 and 90 days after planting, the number of leaves per cutting was counted and their mean was used.

\section{Leaf area $\left(\mathrm{cm}^{2}\right)$}

Leaf area calculated by collecting 4 representative leaves for each treatment from each replication were selected. The area of leaves measured on automatic leaf area meter. The average leaf area was calculated and is presented in $\mathrm{cm}^{2}$

\section{Results and Discussion}

The results obtained during the experiment were recorded and analysed statistically, presented under appropriate headings, subheadings and discussed with available literature.

\section{Growth parameters}

\section{Number of days for sprouting}

An experiment was conducted to optimize the diameter of cutting (D), length of cutting (L) 
and to evaluate the efficacy of IBA concentrations (T) as well as their interactions on number of days for sprouting are presented in Table 1.

\section{Effect of cutting diameter (D)}

The perusal of data in Table 1 reveals that, significantly minimum days for sprouting (8.91) were recorded in $\mathrm{D}_{2}$ i.e. $(1.5 \mathrm{~cm})$ and maximum days (9.25) were observed in $\mathrm{D}_{1}$ i.e. (1 $\mathrm{cm}$ diameter). This might be due to better physiological maturity of the stem and might be due to better translocation of photosynthates (Pooja et al., 2013)

\section{Effect of cutting length (L)}

The data do not show significant effect of cutting length on days required for sprouting.

\section{IBA concentration $(T)$}

Significantly minimum days for sprouting of pomegranate cuttings (8.68) were observed in treatment $\mathrm{T}_{1}$ i.e. IBA $3000 \mathrm{ppm}$ and maximum days (9.40) were observed in treatment $\mathrm{T}_{2}$ i.e. $4000 \mathrm{ppm}$.

This might be due to better role of IBA for hydrolysis and translocation of carbohydrates and nitrogenous substances at the base of cuttings and resulted in accelerated cell division and cell elongation. These results are in accordance with the findings of Shukla et al., (2010), Sinha et al., (2014) and Singh et al., (2014).

\section{Interaction effect of $(\mathrm{L} \times \mathrm{D})$}

The interaction of length and diameter of cutting shows significant effect on days for sprouting. Significantly lowest days (8.64) for sprouting of cutting were observed in interaction of $\mathrm{L}_{3} \mathrm{D}_{2}$ i.e. (length $20 \mathrm{~cm}$ and diameter $1.5 \mathrm{~cm}$ ) which was statistically at par with $\mathrm{L}_{1} \mathrm{D}_{2}$ (8.97) and $\mathrm{L}_{2} \mathrm{D}_{2}$ (9.11). However, highest days (9.28) were observed in interaction of $\mathrm{L}_{1} \mathrm{D}_{1}$.

\section{Interaction effect of $(\mathbf{T} \times \mathbf{D})$}

Significantly lowest days (8.31) for sprouting of pomegranate cuttings was observed in interaction of $\mathrm{T}_{1} \mathrm{D}_{2}$ i.e. (IBA $3000 \mathrm{ppm}$ and 1.5 $\mathrm{cm}$ diameter) and highest days (9.44) were observed in interaction of $\mathrm{T}_{2} \mathrm{D}_{1}$ i.e. (IBA 4000ppm and $1 \mathrm{~cm}$ diameter).

\section{Interaction effect of $(\mathbf{T} \times \mathbf{L})$}

The interaction effect of length of cutting and IBA concentration on days for sprouting shows significant variation. The interaction $\mathrm{T}_{1} \mathrm{~L}_{3}$ recorded significantly lowest days (8.36) for sprouting of cuttings which was statistically at par with interactions of $\mathrm{T}_{1} \mathrm{~L}_{1}$ (8.83 days), $\mathrm{T}_{1} \mathrm{~L}_{3}$ (8.86 days) and highest days (9.53) for sprouting of cuttings were observed in $\mathrm{T}_{2} \mathrm{~L}_{2}$. However, the interaction of diameter, length of cuttings and IBA concentrations $(\mathrm{T} \times \mathrm{D} \times \mathrm{L})$ do not show significant variation on days for sprouting of pomegranate cuttings.

\section{Sprouting percentage}

The effect of diameter of cutting (D), length of cutting (L), IBA concentrations (T) and their interactions on sprouting percentage of pomegranate cuttings are presented in Table 1.

\section{Effect of cutting diameter (D)}

The perusal of data in Table 1 reveals that, significantly highest sprouting percentage $(73.35 \%)$ was recorded in $\mathrm{D}_{2}$ i.e. $(1.5 \mathrm{~cm})$ whereas lowest sprouting percentage (68.03 $\%$ ) was observed in $\mathrm{D}_{1}$ i.e. (1 $\mathrm{cm}$ diameter). This might be due to better physiological maturity of the stem and might be due to better translocation of photosynthates (Pooja et al., 2013). 


\section{Effect of cutting length $(\mathrm{L})$}

The results clearly indicated that significantly highest sprouting percentage $(73.75 \%)$ was recorded in $\mathrm{L}_{3}$ i.e. (20 cm length) however lowest $(71.19 \%)$ was observed in $\mathrm{L}_{2}$ i.e. (15 cm length).

This might be due to highest carbohydrates present in cuttings which are utilized for sprouting. Similarly, another reason might be length of cutting, on which maximum buds are present and later on they sprout (Kaur and Kaur, 2018). These results are in accordance with the findings of Pooja et al., (2013).

\section{IBA concentration $(T)$}

It was evident from the data that, the IBA concentrations significantly affected on sprouting percentage.

The highest sprouting percentage (73.02\%) was observed in $T_{1}$ with the application of IBA 3000 ppm.

While the lowest sprouting percentage (68.88 $\%$ ) was recorded in $\mathrm{T}_{3}$ i.e. IBA $4000 \mathrm{ppm}$. Significantly highest sprouting percentage was due to better utilization of carbohydrates with the help of IBA (Singh et al., 2015).

\section{Interaction effect of $(\mathbf{L} \times \mathbf{D})$}

The interaction of length and diameter of cutting shows significant effect on sprouting percentage. Significantly highest sprouting percentage $(78.88 \%)$ was observed in interaction of $\mathrm{L}_{3} \mathrm{D}_{2}$ i.e. (length $20 \mathrm{~cm}$ and diameter $1.5 \mathrm{~cm})$ and lowest $(68.27 \%)$ was observed in interaction of $\mathrm{L}_{3} \mathrm{D}_{1}$ i.e. (length 20 $\mathrm{cm}$ and diameter $1 \mathrm{~cm}$ ).

\section{Interaction effect of $(T \times D)$}

Significantly highest sprouting percentage $(75.77 \%)$ of pomegranate cuttings was observed in interaction of $\mathrm{T}_{1} \mathrm{D}_{2}$ i.e. (IBA 3000 ppm and $1.5 \mathrm{~cm}$ diameter) followed by interactions of $\mathrm{T}_{3} \mathrm{D}_{2}(74.72 \%)$ and $\mathrm{T}_{2} \mathrm{D}_{2}$ (70.73 \%). However lowest sprouting percentage $(63.44 \%)$ was observed in interaction of $\mathrm{T}_{3} \mathrm{D}_{1}$.

\section{Interaction effect of $(T \times L)$}

The interaction effect of length of cutting and IBA concentration on sprouting percentage shows significant variation. The interaction of $\mathrm{T}_{1} \mathrm{~L}_{3}$ i.e. (IBA $3000 \mathrm{ppm}$ and $20 \mathrm{~cm}$ length) was recorded significantly highest sprouting percentage $(78.33 \%)$ which was statistically at par with interactions of $\mathrm{T}_{2} \mathrm{~L}_{3}(75.78 \%)$ and $\mathrm{T}_{1} \mathrm{~L}_{2}(72.00 \%)$. However, lowest sprouting percentage $(65.00 \%)$ was observed in $\mathrm{T}_{2} \mathrm{~L}_{1}$ i.e. (4000 ppm IBA and $10 \mathrm{~cm}$ length). The interaction of IBA concentrations, diameter and length of cuttings $(\mathrm{T} \times \mathrm{D} \times \mathrm{L})$ on sprouting percentage was found to be non significant.

\section{Number of branches per cutting at 30DAP}

The number of branches per cutting was influenced by diameter of cutting (D), length of cutting (L), IBA concentrations (T) and their interactions presented in Table 2.

\section{Effect of cutting diameter (D)}

The result obtained from data shows non significant effect of cutting diameter on number of branches per cutting.

\section{Effect of cutting length (L)}

The perusal of data in Table 2 reveals that, significantly maximum number of branches per cutting (3.75) was recorded in $\mathrm{L}_{3}$ i.e. $(20$ cm length) and minimum (2.34) was observed in $\mathrm{L}_{1}$ i.e. (10 cm length). This might be due to highest nodes present on cutting and maximum available food material in thick cuttings which is used for initiation and 
growth of sprouts. Similar results were also reported by Jadhav et al., (2003).

\section{IBA concentration ( $T)$}

Significantly maximum number of branches (3.54) for pomegranate cutting was observed in $\mathrm{T}_{1}$ i.e. (IBA $3000 \mathrm{ppm}$ ) while minimum number of branches (2.70) were observed in $\mathrm{T}_{3}$ i.e. (IBA $5000 \mathrm{ppm}$ ). This might be due to the fact that, IBA affect on cell division in the cambium and cell expansion resulting in increasing number of shoots or branches. Similar results were also reported by Thimann (1969), Devi et al., (2016) and Kamboj (2017).

\section{Interaction effect of $(\mathrm{L} \times \mathrm{D})$}

The interaction of length and diameter of cutting shows significant effect on number of branches. Significantly maximum number of branches per cutting (4.08) was observed in interaction of $\mathrm{L}_{3} \mathrm{D}_{2}$ i.e. (length $20 \mathrm{~cm}$ and diameter $1.5 \mathrm{~cm}$ ), while minimum number of branches (2.11) was observed in interaction of $\mathrm{L}_{1} \mathrm{D}_{2}$ i.e. (length $10 \mathrm{~cm}$ and $1.5 \mathrm{~cm}$ diameter).

\section{Interaction effect of $(T \times D)$}

Significantly maximum number of branches of pomegranate cuttings (3.71) was observed in interaction of $\mathrm{T}_{1} \mathrm{D}_{2}$ i.e. (IBA $3000 \mathrm{ppm}$ and $1.5 \mathrm{~cm}$ diameter) which was followed by $\mathrm{T}_{1} \mathrm{D}_{1}$ (3.37), while minimum number of branches (2.66) was observed in interaction of $\mathrm{T}_{2} \mathrm{D}_{1}$ i.e. (IBA $4000 \mathrm{ppm}$ and $1 \mathrm{~cm}$ diameter).

\section{Interaction effect of $(\mathrm{T} \times \mathrm{L})$}

The interaction effect of length of cutting and IBA concentration on number of branches was found to be significant. The interaction of $\mathrm{T}_{1} \mathrm{~L}_{3}$ i.e. (IBA $3000 \mathrm{ppm}$ and $20 \mathrm{~cm}$ length) was recorded significantly maximum number of branches (4.33) and minimum number of branches per cutting (1.93) was observed in $\mathrm{T}_{2} \mathrm{~L}_{1}$. However, the interaction of IBA concentrations, diameter and length of cuttings $(\mathrm{T} \times \mathrm{D} \times \mathrm{L})$ was found to be non significant on number of branches per cutting.

\section{Number of branches per cutting at 60 DAP}

Number of branches per cutting at 60 DAP was influenced by diameter of cutting (D), length of cutting (L), IBA concentrations (T) and their interactions are presented in Table 2.

\section{Effect of cutting diameter (D)}

The data shows non significant effect of cutting diameter on number of branches per cutting.

\section{Effect of cutting length ( $L)$}

Significantly maximum number of branches per cutting (4.22) was recorded in $\mathrm{L}_{3}$ i.e. (20 cm length), and minimum (2.73) was observed in $\mathrm{L}_{1}$. i.e. (10 $\mathrm{cm}$ length). This might be due to highest nodes present on cutting and maximum available food material in thick cuttings which is used for initiation and growth of sprouts. Similar results were also reported by Jadhav et al., (2003).

\section{IBA concentration ( $T)$}

Significantly highest number of branches of pomegranate cuttings (3.78) was observed in treatment $\mathrm{T}_{1}$ i.e. (IBA 3000 ppm) and minimum number of branches (3.08) was observed in $\mathrm{T}_{3}$ i.e. (IBA $5000 \mathrm{ppm}$ ). This might be due to the fact that, IBA affect on cell division in the cambium and cell expansion resulting in increasing number of shoots or branches. Similar results were also reported by Thimann (1969), Devi et al., (2016) and Kamboj (2017). 
Table.1

Factors:

Factor A: Length of cutting

Factor B: Diameter of cutting
Factor C:

IBA concentration

\begin{tabular}{l|l}
$\mathrm{T}_{1}:$ & $3000 \mathrm{ppm}$
\end{tabular}

$\mathrm{T}_{2}: \quad 4000 \mathrm{ppm}$

$\mathrm{T}_{3}: \quad 5000 \mathrm{ppm}$

Table.2 Effect of cutting size and IBA concentration on sprouting of pomegranate cuttings

\begin{tabular}{|c|c|c|}
\hline \multirow[t]{2}{*}{ Treatments } & $\begin{array}{c}\text { Days for } \\
\text { sprouting }\end{array}$ & Sprouting percentage \\
\hline & Days & Percentage \\
\hline \multicolumn{3}{|c|}{ Diameter of cutting (D) } \\
\hline $\mathrm{D}_{1}$ & 9.25 & $68.03 \quad(43.25)$ \\
\hline $\mathrm{D}_{2}$ & 8.91 & $73.35 \quad(47.74)$ \\
\hline S.E N \pm & 0.10 & 1.03 \\
\hline C.D. at $5 \%$ & 0.28 & 3.09 \\
\hline \multicolumn{3}{|c|}{ Length of cutting (L) } \\
\hline $\mathrm{L}_{1}$ & 9.13 & $67.47 \quad(42.84)$ \\
\hline $\mathrm{L}_{2}$ & 9.17 & $71.19 \quad(45.50)$ \\
\hline $\mathrm{L}_{3}$ & 8.93 & $73.75 \quad(48.14)$ \\
\hline S.E N \pm & 0.12 & 1.15 \\
\hline C.D. at $5 \%$ & NS & 3.45 \\
\hline \multicolumn{3}{|c|}{ IBA concentration $(\mathrm{T})$} \\
\hline $\mathrm{T}_{1}$ & 8.68 & $73.02 \quad(47.41)$ \\
\hline $\mathrm{T}_{2}$ & 9.40 & $70.50 \quad(45.17)$ \\
\hline $\mathrm{T}_{3}$ & 9.15 & $68.88 \quad(43.90)$ \\
\hline S.E N \pm & 0.12 & 1.15 \\
\hline C.D. at $5 \%$ & 0.34 & 3.45 \\
\hline \multicolumn{3}{|c|}{ Interaction $(\mathrm{L} \times \mathrm{D})$} \\
\hline $\mathrm{L}_{1} \mathrm{D}_{1}$ & 9.28 & $64.38 \quad(40.42)$ \\
\hline $\mathrm{L}_{2} \mathrm{D}_{1}$ & 9.24 & $71.11 \quad(45.45)$ \\
\hline $\mathrm{L}_{3} \mathrm{D}_{1}$ & 9.22 & $68.27 \quad(43.87)$ \\
\hline $\mathrm{L}_{1} \mathrm{D}_{2}$ & 8.97 & $70.55 \quad(45.25)$ \\
\hline $\mathrm{L}_{2} \mathrm{D}_{2}$ & 9.11 & $71.27 \quad(45.55)$ \\
\hline $\mathrm{L}_{3} \mathrm{D}_{2}$ & 8.64 & $78.88 \quad(52.41)$ \\
\hline S.E N \pm & 0.17 & 1.18 \\
\hline C.D. at $5 \%$ & 0.48 & 3.54 \\
\hline \multicolumn{3}{|c|}{ Interaction (T x D) } \\
\hline $\mathrm{T}_{1} \mathrm{D}_{1}$ & 9.07 & $70.27 \quad(44.96)$ \\
\hline $\mathrm{T}_{1} \mathrm{D}_{2}$ & 8.31 & $75.77 \quad(49.86)$ \\
\hline $\mathrm{T}_{2} \mathrm{D}_{1}$ & 9.44 & $70.27 \quad(45.10)$ \\
\hline $\mathrm{T}_{2} \mathrm{D}_{2}$ & 9.35 & $70.73 \quad(45.24)$ \\
\hline $\mathrm{T}_{3} \mathrm{D}_{1}$ & 9.24 & $63.44 \quad(39.69)$ \\
\hline $\mathrm{T}_{3} \mathrm{D}_{2}$ & 9.06 & $74.72 \quad(48.11)$ \\
\hline S.E N \pm & 0.17 & 1.18 \\
\hline C.D. at $5 \%$ & 0.48 & 3.55 \\
\hline
\end{tabular}

* figures in parenthesis indicate the arc sine values. 


\begin{tabular}{|c|c|c|}
\hline Interaction $(\mathrm{L} \times \mathrm{T})$ & Days & Percentage \\
\hline $\mathrm{T}_{1} \mathrm{~L}_{1}$ & 8.83 & $68.75 \quad(43.92)$ \\
\hline $\mathrm{T}_{1} \mathrm{~L}_{2}$ & 8.86 & $72.00 \quad(46.19)$ \\
\hline $\mathrm{T}_{1} \mathrm{~L}_{3}$ & 8.36 & $78.33 \quad(52.12)$ \\
\hline $\mathrm{T}_{2} \mathrm{~L}_{1}$ & 9.33 & $65.00 \quad(41.08)$ \\
\hline $\mathrm{T}_{2} \mathrm{~L}_{2}$ & 9.53 & $71.08 \quad(45.39)$ \\
\hline $\mathrm{T}_{2} \mathrm{~L}_{3}$ & 9.33 & $75.58 \quad(49.04)$ \\
\hline $\mathrm{T}_{3} \mathrm{~L}_{1}$ & 9.23 & $68.66 \quad(43.51)$ \\
\hline $\mathrm{T}_{3} \mathrm{~L}_{2}$ & 9.13 & $70.50 \quad(44.92)$ \\
\hline $\mathrm{T}_{3} \mathrm{~L}_{3}$ & 9.10 & $67.50 \quad(43.27)$ \\
\hline S.E N \pm & 0.21 & 1.04 \\
\hline C.D. at $5 \%$ & 0.59 & 3.12 \\
\hline \multicolumn{3}{|l|}{ Interaction $(\mathrm{T} \times \mathrm{D} \times \mathrm{L})$} \\
\hline $\mathrm{T}_{1} \mathrm{D}_{1} \mathrm{~L}_{1}$ & 9.06 & $64.16 \quad(40.12)$ \\
\hline $\mathrm{T}_{1} \mathrm{D}_{1} \mathrm{~L}_{2}$ & 9.00 & $73.33 \quad(47.34)$ \\
\hline $\mathrm{T}_{1} \mathrm{D}_{1} \mathrm{~L}_{3}$ & 9.13 & $62.50 \quad(38.88)$ \\
\hline $\mathrm{T}_{1} \mathrm{D}_{2} \mathrm{~L}_{1}$ & 8.60 & $73.85 \quad(47.72)$ \\
\hline $\mathrm{T}_{1} \mathrm{D}_{2} \mathrm{~L}_{2}$ & 8.73 & $70.66 \quad(45.03)$ \\
\hline $\mathrm{T}_{1} \mathrm{D}_{2} \mathrm{~L}_{3}$ & 7.60 & $83.16 \quad(56.84)$ \\
\hline $\mathrm{T}_{2} \mathrm{D}_{1} \mathrm{~L}_{1}$ & 9.53 & $64.16 \quad(40.59)$ \\
\hline $\mathrm{T}_{2} \mathrm{D}_{1} \mathrm{~L}_{2}$ & 9.40 & $70.00 \quad(44.51)$ \\
\hline $\mathrm{T}_{2} \mathrm{D}_{1} \mathrm{~L}_{3}$ & 9.40 & $76.83 \quad(50.20)$ \\
\hline $\mathrm{T}_{2} \mathrm{D}_{2} \mathrm{~L}_{1}$ & 9.13 & $65.83 \quad(41.56)$ \\
\hline $\mathrm{T}_{2} \mathrm{D}_{2} \mathrm{~L}_{2}$ & 9.66 & $72.16 \quad(46.28)$ \\
\hline $\mathrm{T}_{2} \mathrm{D}_{2} \mathrm{~L}_{3}$ & 9.26 & $74.33 \quad(47.87)$ \\
\hline $\mathrm{T}_{3} \mathrm{D}_{1} \mathrm{~L}_{1}$ & 9.26 & $64.83 \quad(40.54)$ \\
\hline $\mathrm{T}_{3} \mathrm{D}_{1} \mathrm{~L}_{2}$ & 9.33 & $70.00 \quad(44.51)$ \\
\hline $\mathrm{T}_{3} \mathrm{D}_{1} \mathrm{~L}_{3}$ & 9.13 & $55.50 \quad(34.01)$ \\
\hline $\mathrm{T}_{3} \mathrm{D}_{2} \mathrm{~L}_{1}$ & 9.20 & $72.50 \quad(46.48)$ \\
\hline $\mathrm{T}_{3} \mathrm{D}_{2} \mathrm{~L}_{2}$ & 8.93 & $71.00 \quad(45.33)$ \\
\hline $\mathrm{T}_{3} \mathrm{D}_{2} \mathrm{~L}_{3}$ & 9.06 & $79.66 \quad(52.52)$ \\
\hline S.E N \pm & 0.30 & 4.30 \\
\hline $\mathrm{CD}$ at $5 \%$ & NS & NS \\
\hline \multicolumn{3}{|c|}{ Treatment details } \\
\hline \multirow[t]{2}{*}{$\mathrm{D}_{1}$-Diameter $1 \mathrm{~cm}$} & $\mathrm{~L}_{1}$ - Length $10 \mathrm{~cm}$ & $\mathrm{~T}_{1}-3000 \mathrm{ppm} \mathrm{IBA}$ \\
\hline & $\mathrm{L}_{2}$ - Length $15 \mathrm{~cm}$ & $\mathrm{~T}_{2}-4000 \mathrm{ppm} \mathrm{IBA}$ \\
\hline $\mathrm{D}_{2}$-Dimeter $1.5 \mathrm{~cm}$ & $\mathrm{~L}_{3}$ - Length $20 \mathrm{~cm}$ & $\mathrm{~T}_{3}-5000 \mathrm{ppm} \mathrm{IBA}$ \\
\hline
\end{tabular}

* figures in parenthesis indicate the arc sine values. 
Table.3 Effect of cutting size and IBA concentration on number of branches per cutting in pomegranate

\begin{tabular}{|c|c|c|c|}
\hline \multirow[t]{2}{*}{ Treatments } & \multicolumn{3}{|c|}{ Number of branches per cutting } \\
\hline & 30DAP & 60DAP & 90DAP \\
\hline \multicolumn{4}{|c|}{$\begin{array}{l}\text { Diameter of cutting } \\
\text { (D) }\end{array}$} \\
\hline$D_{1}$ & 2.93 & 3.33 & 3.47 \\
\hline $\mathrm{D}_{2}$ & 3.14 & 3.57 & 3.70 \\
\hline S.E N \pm & 0.11 & 0.11 & 0.13 \\
\hline C.D. at $5 \%$ & NS & NS & NS \\
\hline \multicolumn{4}{|c|}{ Length of cutting (L) } \\
\hline $\mathrm{L}_{1}$ & 2.34 & 2.73 & 3.17 \\
\hline $\mathrm{L}_{2}$ & 3.01 & 3.51 & 3.36 \\
\hline $\mathrm{L}_{3}$ & 3.75 & 4.22 & 4.22 \\
\hline S.E N \pm & 0.13 & 0.13 & 0.16 \\
\hline C.D. at $5 \%$ & 0.37 & 0.37 & 0.44 \\
\hline \multicolumn{4}{|c|}{ IBA concentration $(\mathrm{T})$} \\
\hline $\mathrm{T}_{1}$ & 3.54 & 3.78 & 4.12 \\
\hline $\mathrm{T}_{2}$ & 2.78 & 3.48 & 3.48 \\
\hline$T_{3}$ & 2.70 & 3.08 & 3.15 \\
\hline S.E N \pm & 0.13 & 0.13 & 0.16 \\
\hline C.D. at $5 \%$ & 0.38 & NS & 0.44 \\
\hline \multicolumn{4}{|c|}{ Interaction (L x D) } \\
\hline $\mathrm{L}_{1} \mathrm{D}_{1}$ & 2.57 & 2.91 & 3.26 \\
\hline $\mathrm{L}_{2} \mathrm{D}_{1}$ & 2.80 & 3.26 & 3.24 \\
\hline $\mathrm{L}_{3} \mathrm{D}_{1}$ & 3.42 & 3.82 & 3.91 \\
\hline $\mathrm{L}_{1} \mathrm{D}_{2}$ & 2.11 & 2.55 & 3.08 \\
\hline $\mathrm{L}_{2} \mathrm{D}_{2}$ & 3.22 & 3.75 & 3.48 \\
\hline $\mathrm{L}_{3} \mathrm{D}_{2}$ & 4.08 & 4.42 & 4.53 \\
\hline S.E N \pm & 0.19 & 0.19 & 0.22 \\
\hline C.D. at $5 \%$ & 0.53 & 0.52 & 0.62 \\
\hline \multicolumn{4}{|c|}{ Interaction ( $\mathrm{T} \times \mathrm{D})$} \\
\hline $\mathrm{T}_{1} \mathrm{D}_{1}$ & 3.37 & 3.51 & 3.75 \\
\hline $\mathrm{T}_{1} \mathrm{D}_{2}$ & 3.71 & 4.06 & 4.48 \\
\hline $\mathrm{T}_{2} \mathrm{D}_{1}$ & 2.66 & 3.48 & 3.55 \\
\hline $\mathrm{T}_{2} \mathrm{D}_{2}$ & 2.91 & 3.48 & 3.42 \\
\hline $\mathrm{T}_{3} \mathrm{D}_{1}$ & 2.75 & 3.00 & 3.11 \\
\hline $\mathrm{T}_{3} \mathrm{D}_{2}$ & 2.80 & 3.17 & 3.20 \\
\hline S.E N \pm & 0.19 & 0.19 & 0.22 \\
\hline C.D. at $5 \%$ & 0.53 & 0.52 & 0.62 \\
\hline
\end{tabular}




\begin{tabular}{|c|c|c|c|}
\hline Interaction $(\mathrm{L} \times \mathrm{T})$ & 30DAP & 60DAP & 90DAP \\
\hline $\mathrm{T}_{1} \mathrm{~L}_{1}$ & 2.93 & 3.26 & 3.63 \\
\hline $\mathrm{T}_{1} \mathrm{~L}_{2}$ & 3.36 & 3.43 & 3.56 \\
\hline $\mathrm{T}_{1} \mathrm{~L}_{3}$ & 4.33 & 4.66 & 5.16 \\
\hline $\mathrm{T}_{2} \mathrm{~L}_{1}$ & 1.93 & 2.63 & 2.83 \\
\hline $\mathrm{T}_{2} \mathrm{~L}_{2}$ & 2.96 & 3.66 & 3.60 \\
\hline $\mathrm{T}_{2} \mathrm{~L}_{3}$ & 3.46 & 4.16 & 4.03 \\
\hline $\mathrm{T}_{3} \mathrm{~L}_{1}$ & 2.16 & 2.30 & 3.06 \\
\hline $\mathrm{T}_{3} \mathrm{~L}_{2}$ & 2.70 & 3.43 & 2.93 \\
\hline $\mathrm{T}_{3} \mathrm{~L}_{3}$ & 3.46 & 3.53 & 3.46 \\
\hline S.E N \pm & 0.23 & 0.23 & 0.27 \\
\hline C.D. at $5 \%$ & 0.65 & 0.64 & 0.81 \\
\hline Interaction $(T \times D \times L)$ & & & \\
\hline $\mathrm{T}_{1} \mathrm{D}_{1} \mathrm{~L}_{1}$ & 3.33 & 3.20 & 3.26 \\
\hline $\mathrm{T}_{1} \mathrm{D}_{1} \mathrm{~L}_{2}$ & 3.13 & 3.13 & 3.40 \\
\hline $\mathrm{T}_{1} \mathrm{D}_{1} \mathrm{~L}_{3}$ & 3.66 & 4.20 & 4.60 \\
\hline $\mathrm{T}_{1} \mathrm{D}_{2} \mathrm{~L}_{1}$ & 2.53 & 3.33 & 4.00 \\
\hline $\mathrm{T}_{1} \mathrm{D}_{2} \mathrm{~L}_{2}$ & 3.60 & 3.73 & 3.73 \\
\hline $\mathrm{T}_{1} \mathrm{D}_{2} \mathrm{~L}_{3}$ & 5.00 & 5.13 & 5.73 \\
\hline $\mathrm{T}_{2} \mathrm{D}_{1} \mathrm{~L}_{1}$ & 2.20 & 3.06 & 3.33 \\
\hline $\mathrm{T}_{2} \mathrm{D}_{1} \mathrm{~L}_{2}$ & 2.46 & 3.40 & 3.20 \\
\hline $\mathrm{T}_{2} \mathrm{D}_{1} \mathrm{~L}_{3}$ & 3.33 & 4.00 & 4.13 \\
\hline $\mathrm{T}_{2} \mathrm{D}_{2} \mathrm{~L}_{1}$ & 1.66 & 2.20 & 2.33 \\
\hline $\mathrm{T}_{2} \mathrm{D}_{2} \mathrm{~L}_{2}$ & 3.46 & 3.93 & 4.00 \\
\hline $\mathrm{T}_{2} \mathrm{D}_{2} \mathrm{~L}_{3}$ & 3.60 & 4.33 & 3.93 \\
\hline $\mathrm{T}_{3} \mathrm{D}_{1} \mathrm{~L}_{1}$ & 2.20 & 2.46 & 3.20 \\
\hline $\mathrm{T}_{3} \mathrm{D}_{1} \mathrm{~L}_{2}$ & 2.80 & 3.26 & 3.13 \\
\hline $\mathrm{T}_{3} \mathrm{D}_{1} \mathrm{~L}_{3}$ & 3.26 & 3.26 & 3.00 \\
\hline $\mathrm{T}_{3} \mathrm{D}_{2} \mathrm{~L}_{1}$ & 2.13 & 2.13 & 2.93 \\
\hline $\mathrm{T}_{3} \mathrm{D}_{2} \mathrm{~L}_{2}$ & 2.60 & 3.60 & 2.73 \\
\hline $\mathrm{T}_{3} \mathrm{D}_{2} \mathrm{~L}_{3}$ & 3.66 & 3.80 & 3.93 \\
\hline S.E N \pm & 0.33 & 0.33 & 0.39 \\
\hline $\mathrm{CD}$ at $5 \%$ & NS & 0.91 & NS \\
\hline \multicolumn{4}{|c|}{ Treatment details } \\
\hline \multirow[t]{2}{*}{$\mathrm{D}_{1}$-Diameter $1 \mathrm{~cm}$} & \multirow{2}{*}{\multicolumn{2}{|c|}{$\begin{array}{l}\mathrm{L}_{1}-\text { Length } 10 \mathrm{~cm} \\
\mathrm{~L}_{2} \text { - Length } 15 \mathrm{~cm}\end{array}$}} & \\
\hline & & & $\mathrm{T}_{2}-4000 \mathrm{ppm}$ IBA \\
\hline $\mathrm{D}_{2}$-Dimeter $1.5 \mathrm{~cm}$ & $\mathrm{~L}_{3}$ - Length $20 \mathrm{~cm}$ & \multicolumn{2}{|c|}{$\mathrm{T}_{3}-5000 \mathrm{ppm} \mathrm{IBA}$} \\
\hline
\end{tabular}


Table.4 Effect of cutting size and IBA concentration on shoot length of pomegranate cuttings

\begin{tabular}{|c|c|c|}
\hline \multirow[t]{2}{*}{ Treatments } & \multicolumn{2}{|c|}{ Shoot length $(\mathrm{cm})$} \\
\hline & 60DAP & 90DAP \\
\hline \multicolumn{3}{|c|}{ Diameter of cutting (D) } \\
\hline $\mathrm{D}_{1}$ & 13.34 & 17.14 \\
\hline $\mathrm{D}_{2}$ & 14.09 & 17.83 \\
\hline S.E N \pm & 0.32 & 0.49 \\
\hline C.D. at $5 \%$ & NS & NS \\
\hline \multicolumn{3}{|c|}{ Length of cutting (L) } \\
\hline $\mathrm{L}_{1}$ & 13.30 & 16.54 \\
\hline $\mathrm{L}_{2}$ & 12.91 & 17.64 \\
\hline $\mathrm{L}_{3}$ & 15.08 & 18.26 \\
\hline S.E N \pm & 0.40 & 0.60 \\
\hline C.D. at $5 \%$ & 1.10 & 1.68 \\
\hline \multicolumn{3}{|c|}{ IBA concentration ( $\mathrm{T}$ ) } \\
\hline $\mathrm{T}_{1}$ & 15.38 & 18.25 \\
\hline $\mathrm{T}_{2}$ & 13.04 & 17.71 \\
\hline $\mathrm{T}_{3}$ & 12.86 & 16.48 \\
\hline S.E N \pm & 0.40 & 0.60 \\
\hline C.D. at $5 \%$ & 1.10 & 1.68 \\
\hline \multicolumn{3}{|c|}{ Interaction $(\mathrm{L} \times \mathrm{D})$} \\
\hline $\mathrm{L}_{1} \mathrm{D}_{1}$ & 13.55 & 16.28 \\
\hline $\mathrm{L}_{2} \mathrm{D}_{1}$ & 12.35 & 17.77 \\
\hline $\mathrm{L}_{3} \mathrm{D}_{1}$ & 14.40 & 17.35 \\
\hline $\mathrm{L}_{1} \mathrm{D}_{2}$ & 13.04 & 16.80 \\
\hline $\mathrm{L}_{2} \mathrm{D}_{2}$ & 13.46 & 17.51 \\
\hline $\mathrm{L}_{3} \mathrm{D}_{2}$ & 15.77 & 19.17 \\
\hline S.E N \pm & 0.56 & 0.85 \\
\hline C.D. at $5 \%$ & 1.56 & 2.37 \\
\hline \multicolumn{3}{|c|}{ Interaction $(\mathrm{T} \times \mathrm{D})$} \\
\hline $\mathrm{T}_{1} \mathrm{D}_{1}$ & 13.91 & 17.11 \\
\hline $\mathrm{T}_{1} \mathrm{D}_{2}$ & 16.86 & 19.40 \\
\hline $\mathrm{T}_{2} \mathrm{D}_{1}$ & 13.51 & 18.33 \\
\hline $\mathrm{T}_{2} \mathrm{D}_{2}$ & 12.57 & 17.08 \\
\hline $\mathrm{T}_{3} \mathrm{D}_{1}$ & 12.88 & 15.97 \\
\hline $\mathrm{T}_{3} \mathrm{D}_{2}$ & 12.84 & 17.00 \\
\hline S.E N \pm & 0.56 & 0.85 \\
\hline C.D. at $5 \%$ & 1.56 & 2.37 \\
\hline
\end{tabular}




\begin{tabular}{|c|c|c|}
\hline Interaction $(\mathrm{L} \times \mathrm{T})$ & 60DAP & 90DAP \\
\hline $\mathrm{T}_{1} \mathrm{~L}_{1}$ & 14.40 & 15.86 \\
\hline $\mathrm{T}_{1} \mathrm{~L}_{2}$ & 15.53 & 18.70 \\
\hline $\mathrm{T}_{1} \mathrm{~L}_{3}$ & 16.23 & 20.20 \\
\hline $\mathrm{T}_{2} \mathrm{~L}_{1}$ & 13.03 & 17.46 \\
\hline $\mathrm{T}_{2} \mathrm{~L}_{2}$ & 11.96 & 17.90 \\
\hline $\mathrm{T}_{2} \mathrm{~L}_{3}$ & 14.13 & 17.76 \\
\hline $\mathrm{T}_{3} \mathrm{~L}_{1}$ & 12.46 & 16.30 \\
\hline $\mathrm{T}_{3} \mathrm{~L}_{2}$ & 11.23 & 16.33 \\
\hline$T_{3} L_{3}$ & 14.90 & 16.83 \\
\hline S.E N \pm & 0.69 & 1.05 \\
\hline C.D. at $5 \%$ & 1.92 & 2.91 \\
\hline \multicolumn{3}{|l|}{ Interaction $(\mathrm{T} \times \mathrm{D} \times \mathrm{L})$} \\
\hline $\mathrm{T}_{1} \mathrm{D}_{1} \mathrm{~L}_{1}$ & 13.06 & 13.80 \\
\hline $\mathrm{T}_{1} \mathrm{D}_{1} \mathrm{~L}_{2}$ & 15.00 & 19.06 \\
\hline $\mathrm{T}_{1} \mathrm{D}_{1} \mathrm{~L}_{3}$ & 13.66 & 18.46 \\
\hline $\mathrm{T}_{1} \mathrm{D}_{2} \mathrm{~L}_{1}$ & 15.73 & 17.93 \\
\hline $\mathrm{T}_{1} \mathrm{D}_{2} \mathrm{~L}_{2}$ & 16.06 & 18.33 \\
\hline $\mathrm{T}_{1} \mathrm{D}_{2} \mathrm{~L}_{3}$ & 18.80 & 21.93 \\
\hline $\mathrm{T}_{2} \mathrm{D}_{1} \mathrm{~L}_{1}$ & 14.66 & 18.60 \\
\hline $\mathrm{T}_{2} \mathrm{D}_{1} \mathrm{~L}_{2}$ & 10.86 & 18.00 \\
\hline $\mathrm{T}_{2} \mathrm{D}_{1} \mathrm{~L}_{3}$ & 15.00 & 18.40 \\
\hline $\mathrm{T}_{2} \mathrm{D}_{2} \mathrm{~L}_{1}$ & 11.40 & 16.33 \\
\hline $\mathrm{T}_{2} \mathrm{D}_{2} \mathrm{~L}_{2}$ & 13.06 & 17.80 \\
\hline $\mathrm{T}_{2} \mathrm{D}_{2} \mathrm{~L}_{3}$ & 13.26 & 17.13 \\
\hline $\mathrm{T}_{3} \mathrm{D}_{1} \mathrm{~L}_{1}$ & 12.93 & 16.46 \\
\hline $\mathrm{T}_{3} \mathrm{D}_{1} \mathrm{~L}_{2}$ & 11.20 & 16.26 \\
\hline $\mathrm{T}_{3} \mathrm{D}_{1} \mathrm{~L}_{3}$ & 14.53 & 15.20 \\
\hline $\mathrm{T}_{3} \mathrm{D}_{2} \mathrm{~L}_{1}$ & 12.00 & 16.13 \\
\hline $\mathrm{T}_{3} \mathrm{D}_{2} \mathrm{~L}_{2}$ & 11.26 & 16.40 \\
\hline $\mathrm{T}_{3} \mathrm{D}_{2} \mathrm{~L}_{3}$ & 15.26 & 18.46 \\
\hline S.E N \pm & 0.98 & 1.48 \\
\hline $\mathrm{CD}$ at $5 \%$ & 2.71 & 4.11 \\
\hline \multicolumn{3}{|c|}{ Treatment details } \\
\hline \multirow[t]{2}{*}{$\mathrm{D}_{1}$-Diameter $1 \mathrm{~cm}$} & $\mathrm{~L}_{1}$ - Length $10 \mathrm{~cm}$ & $\mathrm{~T}_{1}-3000 \mathrm{ppm}$ IBA \\
\hline & $\mathrm{L}_{2}$ - Length $15 \mathrm{~cm}$ & $\mathrm{~T}_{2}-4000 \mathrm{ppm} \mathrm{IBA}$ \\
\hline $\mathrm{D}_{2}$-Dimeter $1.5 \mathrm{~cm}$ & $\mathrm{~L}_{3}$ - Length $20 \mathrm{~cm}$ & $\mathrm{~T}_{3}-5000 \mathrm{ppm} \mathrm{IBA}$ \\
\hline
\end{tabular}


Table.5 Effect of cutting size and IBA concentration on shoot diameter of pomegranate cuttings

\begin{tabular}{|c|c|c|}
\hline \multirow[t]{2}{*}{ Treatments } & \multicolumn{2}{|c|}{ Shoot diameter $(\mathbf{m m})$} \\
\hline & 60DAP & 90DAP \\
\hline \multicolumn{3}{|c|}{ Diameter of cutting (D) } \\
\hline $\mathrm{D}_{1}$ & 1.41 & 1.48 \\
\hline $\mathrm{D}_{2}$ & 1.46 & 1.51 \\
\hline S.E N \pm & 0.016 & 0.028 \\
\hline C.D. at $5 \%$ & NS & NS \\
\hline \multicolumn{3}{|c|}{ Length of cutting (L) } \\
\hline $\mathrm{L}_{1}$ & 1.37 & 1.46 \\
\hline $\mathrm{L}_{2}$ & 1.42 & 1.49 \\
\hline $\mathrm{L}_{3}$ & 1.52 & 1.53 \\
\hline S.E N \pm & 0.019 & 0.034 \\
\hline C.D. at $5 \%$ & NS & NS \\
\hline \multicolumn{3}{|c|}{ IBA concentration $(\mathrm{T})$} \\
\hline $\mathrm{T}_{1}$ & 1.50 & 1.58 \\
\hline $\mathrm{T}_{2}$ & 1.39 & 1.45 \\
\hline $\mathrm{T}_{3}$ & 1.42 & 1.46 \\
\hline S.E N \pm & 0.019 & 0.034 \\
\hline C.D. at $5 \%$ & NS & NS \\
\hline \multicolumn{3}{|c|}{ Interaction $(\mathrm{L} \times \mathrm{D})$} \\
\hline $\mathrm{L}_{1} \mathrm{D}_{1}$ & 1.36 & 1.45 \\
\hline $\mathrm{L}_{2} \mathrm{D}_{1}$ & 1.39 & 1.51 \\
\hline $\mathrm{L}_{3} \mathrm{D}_{1}$ & 1.48 & 1.47 \\
\hline $\mathrm{L}_{1} \mathrm{D}_{2}$ & 1.38 & 1.47 \\
\hline $\mathrm{L}_{2} \mathrm{D}_{2}$ & 1.44 & 1.47 \\
\hline $\mathrm{L}_{3} \mathrm{D}_{2}$ & 1.56 & 1.60 \\
\hline S.E N \pm & 0.028 & 0.049 \\
\hline C.D. at $5 \%$ & 0.077 & NS \\
\hline \multicolumn{3}{|c|}{ Interaction $(\mathrm{T} \times \mathrm{D})$} \\
\hline $\mathrm{T}_{1} \mathrm{D}_{1}$ & 1.42 & 1.51 \\
\hline $\mathrm{T}_{1} \mathrm{D}_{2}$ & 1.59 & 1.65 \\
\hline $\mathrm{T}_{2} \mathrm{D}_{1}$ & 1.43 & 1.50 \\
\hline $\mathrm{T}_{2} \mathrm{D}_{2}$ & 1.35 & 1.41 \\
\hline $\mathrm{T}_{3} \mathrm{D}_{1}$ & 1.39 & 1.43 \\
\hline $\mathrm{T}_{3} \mathrm{D}_{2}$ & 1.45 & 1.49 \\
\hline S.E N \pm & 0.028 & 0.049 \\
\hline C.D. at $5 \%$ & 0.077 & 0.13 \\
\hline
\end{tabular}




\begin{tabular}{|c|c|c|}
\hline Interaction $(\mathrm{L} \times \mathrm{T})$ & 60DAP & 90DAP \\
\hline $\mathrm{T}_{1} \mathrm{~L}_{1}$ & 1.42 & 1.45 \\
\hline $\mathrm{T}_{1} \mathrm{~L}_{2}$ & 1.46 & 1.55 \\
\hline $\mathrm{T}_{1} \mathrm{~L}_{3}$ & 1.62 & 1.74 \\
\hline $\mathrm{T}_{2} \mathrm{~L}_{1}$ & 1.32 & 1.52 \\
\hline $\mathrm{T}_{2} \mathrm{~L}_{2}$ & 1.43 & 1.44 \\
\hline $\mathrm{T}_{2} \mathrm{~L}_{3}$ & 1.41 & 1.40 \\
\hline $\mathrm{T}_{3} \mathrm{~L}_{1}$ & 1.37 & 1.42 \\
\hline $\mathrm{T}_{3} \mathrm{~L}_{2}$ & 1.35 & 1.48 \\
\hline$T_{3} L_{3}$ & 1.53 & 1.47 \\
\hline S.E N \pm & 0.034 & 0.060 \\
\hline C.D. at $5 \%$ & NS & 0.16 \\
\hline \multicolumn{3}{|l|}{ Interaction $(\mathrm{T} \times \mathrm{D} \times \mathrm{L})$} \\
\hline $\mathrm{T}_{1} \mathrm{D}_{1} \mathrm{~L}_{1}$ & 1.34 & 1.41 \\
\hline $\mathrm{T}_{1} \mathrm{D}_{1} \mathrm{~L}_{2}$ & 1.44 & 1.60 \\
\hline $\mathrm{T}_{1} \mathrm{D}_{1} \mathrm{~L}_{3}$ & 1.47 & 1.53 \\
\hline $\mathrm{T}_{1} \mathrm{D}_{2} \mathrm{~L}_{1}$ & 1.51 & 1.49 \\
\hline $\mathrm{T}_{1} \mathrm{D}_{2} \mathrm{~L}_{2}$ & 1.49 & 1.50 \\
\hline $\mathrm{T}_{1} \mathrm{D}_{2} \mathrm{~L}_{3}$ & 1.77 & 1.95 \\
\hline $\mathrm{T}_{2} \mathrm{D}_{1} \mathrm{~L}_{1}$ & 1.44 & 1.60 \\
\hline $\mathrm{T}_{2} \mathrm{D}_{1} \mathrm{~L}_{2}$ & 1.40 & 1.42 \\
\hline $\mathrm{T}_{2} \mathrm{D}_{1} \mathrm{~L}_{3}$ & 1.44 & 1.47 \\
\hline $\mathrm{T}_{2} \mathrm{D}_{2} \mathrm{~L}_{1}$ & 1.20 & 1.43 \\
\hline $\mathrm{T}_{2} \mathrm{D}_{2} \mathrm{~L}_{2}$ & 1.46 & 1.45 \\
\hline $\mathrm{T}_{2} \mathrm{D}_{2} \mathrm{~L}_{3}$ & 1.38 & 1.33 \\
\hline $\mathrm{T}_{3} \mathrm{D}_{1} \mathrm{~L}_{1}$ & 1.31 & 1.34 \\
\hline $\mathrm{T}_{3} \mathrm{D}_{1} \mathrm{~L}_{2}$ & 1.32 & 1.51 \\
\hline $\mathrm{T}_{3} \mathrm{D}_{1} \mathrm{~L}_{3}$ & 1.53 & 1.43 \\
\hline $\mathrm{T}_{3} \mathrm{D}_{2} \mathrm{~L}_{1}$ & 1.44 & 1.50 \\
\hline $\mathrm{T}_{3} \mathrm{D}_{2} \mathrm{~L}_{2}$ & 1.38 & 1.46 \\
\hline $\mathrm{T}_{3} \mathrm{D}_{2} \mathrm{~L}_{3}$ & 1.52 & 1.51 \\
\hline S.E N \pm & 0.048 & 0.085 \\
\hline $\mathrm{CD}$ at $5 \%$ & NS & 0.23 \\
\hline \multicolumn{3}{|c|}{ Treatment details } \\
\hline \multirow{2}{*}{$\mathrm{D}_{1}$-Diameter $1 \mathrm{~cm}$} & $\mathrm{~L}_{1}$ - Length $10 \mathrm{~cm}$ & $\mathrm{~T}_{1^{-}} 3000 \mathrm{ppm}$ IBA \\
\hline & $\mathrm{L}_{2}$ - Length $15 \mathrm{~cm}$ & $\mathrm{~T}_{2}-4000 \mathrm{ppm} \mathrm{IBA}$ \\
\hline $\mathrm{D}_{2}$-Dimeter $1.5 \mathrm{~cm}$ & $\mathrm{~L}_{3}$ - Length $20 \mathrm{~cm}$ & $\mathrm{~T}_{3}-5000 \mathrm{ppm} \mathrm{IBA}$ \\
\hline
\end{tabular}


Table.6 Effect of cutting size and IBA concentration on shoot weight of pomegranate cuttings

\begin{tabular}{|c|c|c|}
\hline Treatments & $\begin{array}{c}\text { Fresh weight of shoot } \\
\text { (g) } \\
\text { 60DAP }\end{array}$ & $\begin{array}{c}\text { Dry weight of shoot } \\
\text { (g) } \\
\text { 90DAP }\end{array}$ \\
\hline \multicolumn{3}{|c|}{ Diameter of cutting (D) } \\
\hline $\mathrm{D}_{1}$ & 4.54 & 1.22 \\
\hline $\mathrm{D}_{2}$ & 5.84 & 1.74 \\
\hline S.E N \pm & 0.029 & 0.058 \\
\hline C.D. at $5 \%$ & 0.082 & 0.16 \\
\hline \multicolumn{3}{|c|}{ Length of cutting (L) } \\
\hline $\mathrm{L}_{1}$ & 4.02 & 1.01 \\
\hline $\mathrm{L}_{2}$ & 5.05 & 1.52 \\
\hline $\mathrm{L}_{3}$ & 6.10 & 1.99 \\
\hline S.E N \pm & 0.036 & 0.072 \\
\hline C.D. at $5 \%$ & 0.10 & 0.19 \\
\hline \multicolumn{3}{|c|}{ IBA concentration $(\mathrm{T})$} \\
\hline $\mathrm{T}_{1}$ & 5.91 & 1.76 \\
\hline $\mathrm{T}_{2}$ & 5.51 & 1.46 \\
\hline $\mathrm{T}_{3}$ & 5.65 & 1.51 \\
\hline S.E N \pm & 0.036 & 0.072 \\
\hline C.D. at $5 \%$ & 0.10 & 0.19 \\
\hline \multicolumn{3}{|c|}{ Interaction $(\mathrm{L} \times \mathrm{D})$} \\
\hline $\mathrm{L}_{1} \mathrm{D}_{1}$ & 4.02 & 0.96 \\
\hline $\mathrm{L}_{2} \mathrm{D}_{1}$ & 5.82 & 1.30 \\
\hline $\mathrm{L}_{3} \mathrm{D}_{1}$ & 6.79 & 1.52 \\
\hline $\mathrm{L}_{1} \mathrm{D}_{2}$ & 4.18 & 1.06 \\
\hline $\mathrm{L}_{2} \mathrm{D}_{2}$ & 5.08 & 1.64 \\
\hline $\mathrm{L}_{3} \mathrm{D}_{2}$ & 7.02 & 1.96 \\
\hline S.E N \pm & 0.051 & 0.10 \\
\hline C.D. at $5 \%$ & 0.14 & 0.28 \\
\hline \multicolumn{3}{|c|}{ Interaction $(\mathrm{T} \times \mathrm{D})$} \\
\hline $\mathrm{T}_{1} \mathrm{D}_{1}$ & 5.74 & 1.36 \\
\hline $\mathrm{T}_{1} \mathrm{D}_{2}$ & 6.88 & 1.86 \\
\hline $\mathrm{T}_{2} \mathrm{D}_{1}$ & 5.77 & 1.23 \\
\hline $\mathrm{T}_{2} \mathrm{D}_{2}$ & 5.26 & 1.39 \\
\hline $\mathrm{T}_{3} \mathrm{D}_{1}$ & 5.12 & 1.19 \\
\hline $\mathrm{T}_{3} \mathrm{D}_{2}$ & 6.17 & 1.41 \\
\hline S.E N \pm & 0.051 & 0.10 \\
\hline C.D. at $5 \%$ & 0.14 & 0.28 \\
\hline
\end{tabular}




\begin{tabular}{|c|c|c|}
\hline Interaction $(\mathrm{L} \times \mathrm{T})$ & 60DAP & 90DAP \\
\hline $\mathrm{T}_{1} \mathrm{~L}_{1}$ & 4.11 & 1.04 \\
\hline $\mathrm{T}_{1} \mathrm{~L}_{2}$ & 5.82 & 1.81 \\
\hline $\mathrm{T}_{1} \mathrm{~L}_{3}$ & 6.80 & 2.42 \\
\hline $\mathrm{T}_{2} \mathrm{~L}_{1}$ & 4.72 & 0.65 \\
\hline $\mathrm{T}_{2} \mathrm{~L}_{2}$ & 5.53 & 2.12 \\
\hline $\mathrm{T}_{2} \mathrm{~L}_{3}$ & 6.29 & 1.51 \\
\hline $\mathrm{T}_{3} \mathrm{~L}_{1}$ & 5.24 & 1.34 \\
\hline $\mathrm{T}_{3} \mathrm{~L}_{2}$ & 5.50 & 1.13 \\
\hline$T_{3} L_{3}$ & 6.13 & 1.44 \\
\hline S.E N \pm & 0.063 & 0.12 \\
\hline C.D. at $5 \%$ & 0.17 & 0.34 \\
\hline \multicolumn{3}{|l|}{ Interaction $(\mathrm{T} \times \mathrm{D} \times \mathrm{L})$} \\
\hline $\mathrm{T}_{1} \mathrm{D}_{1} \mathrm{~L}_{1}$ & 5.59 & 1.52 \\
\hline $\mathrm{T}_{1} \mathrm{D}_{1} \mathrm{~L}_{2}$ & 6.06 & 1.99 \\
\hline $\mathrm{T}_{1} \mathrm{D}_{1} \mathrm{~L}_{3}$ & 5.57 & 1.76 \\
\hline $\mathrm{T}_{1} \mathrm{D}_{2} \mathrm{~L}_{1}$ & 4.63 & 0.56 \\
\hline $\mathrm{T}_{1} \mathrm{D}_{2} \mathrm{~L}_{2}$ & 5.58 & 1.64 \\
\hline $\mathrm{T}_{1} \mathrm{D}_{2} \mathrm{~L}_{3}$ & 8.07 & 3.07 \\
\hline $\mathrm{T}_{2} \mathrm{D}_{1} \mathrm{~L}_{1}$ & 4.37 & 2.06 \\
\hline $\mathrm{T}_{2} \mathrm{D}_{1} \mathrm{~L}_{2}$ & 6.67 & 2.13 \\
\hline $\mathrm{T}_{2} \mathrm{D}_{1} \mathrm{~L}_{3}$ & 6.28 & 2.15 \\
\hline $\mathrm{T}_{2} \mathrm{D}_{2} \mathrm{~L}_{1}$ & 4.07 & 1.00 \\
\hline $\mathrm{T}_{2} \mathrm{D}_{2} \mathrm{~L}_{2}$ & 5.40 & 2.31 \\
\hline $\mathrm{T}_{2} \mathrm{D}_{2} \mathrm{~L}_{3}$ & 6.31 & 2.07 \\
\hline $\mathrm{T}_{3} \mathrm{D}_{1} \mathrm{~L}_{1}$ & 5.12 & 1.06 \\
\hline $\mathrm{T}_{3} \mathrm{D}_{1} \mathrm{~L}_{2}$ & 4.74 & 0.67 \\
\hline $\mathrm{T}_{3} \mathrm{D}_{1} \mathrm{~L}_{3}$ & 5.52 & 1.84 \\
\hline $\mathrm{T}_{3} \mathrm{D}_{2} \mathrm{~L}_{1}$ & 5.37 & 1.61 \\
\hline $\mathrm{T}_{3} \mathrm{D}_{2} \mathrm{~L}_{2}$ & 6.27 & 1.59 \\
\hline $\mathrm{T}_{3} \mathrm{D}_{2} \mathrm{~L}_{3}$ & 6.89 & 2.04 \\
\hline S.E N \pm & 0.089 & 0.17 \\
\hline $\mathrm{CD}$ at $5 \%$ & 0.24 & 0.48 \\
\hline \multicolumn{3}{|c|}{ Treatment details } \\
\hline \multirow{2}{*}{$\mathrm{D}_{1}$-Diameter $1 \mathrm{~cm}$} & $\mathrm{~L}_{1}$ - Length $10 \mathrm{~cm}$ & $\mathrm{~T}_{1^{-}} 3000 \mathrm{ppm}$ IBA \\
\hline & $\mathrm{L}_{2}$ - Length $15 \mathrm{~cm}$ & $\mathrm{~T}_{2}-4000 \mathrm{ppm} \mathrm{IBA}$ \\
\hline $\mathrm{D}_{2}$-Dimeter $1.5 \mathrm{~cm}$ & $\mathrm{~L}_{3}$ - Length $20 \mathrm{~cm}$ & $\mathrm{~T}_{3}-5000 \mathrm{ppm} \mathrm{IBA}$ \\
\hline
\end{tabular}


Table.7 Effect of cutting size and IBA concentration on shoot to root ratio of pomegranate cuttings

\begin{tabular}{|c|c|}
\hline \multirow[t]{2}{*}{ Treatments } & \multirow{2}{*}{$\begin{array}{c}\text { Shoot to root ratio } \\
\text { 90DAP }\end{array}$} \\
\hline & \\
\hline \multicolumn{2}{|c|}{ Diameter of cutting (D) } \\
\hline$D_{1}$ & 1.14 \\
\hline $\mathrm{D}_{2}$ & 1.45 \\
\hline S.E N \pm & 0.045 \\
\hline C.D. at $5 \%$ & 0.12 \\
\hline \multicolumn{2}{|c|}{ Length of cutting (L) } \\
\hline $\mathrm{L}_{1}$ & 1.01 \\
\hline $\mathrm{L}_{2}$ & 1.09 \\
\hline $\mathrm{L}_{3}$ & 1.36 \\
\hline S.E N \pm & 0.055 \\
\hline C.D. at $5 \%$ & 0.15 \\
\hline \multicolumn{2}{|c|}{ IBA concentration $(\mathrm{T})$} \\
\hline $\mathrm{T}_{1}$ & 1.23 \\
\hline $\mathrm{T}_{2}$ & 1.11 \\
\hline $\mathrm{T}_{3}$ & 1.02 \\
\hline S.E N \pm & 0.055 \\
\hline C.D. at $5 \%$ & 0.15 \\
\hline \multicolumn{2}{|c|}{ Interaction $(\mathrm{L} \times \mathrm{D})$} \\
\hline $\mathrm{L}_{1} \mathrm{D}_{1}$ & 0.98 \\
\hline $\mathrm{L}_{2} \mathrm{D}_{1}$ & 1.11 \\
\hline $\mathrm{L}_{3} \mathrm{D}_{1}$ & 1.23 \\
\hline $\mathrm{L}_{1} \mathrm{D}_{2}$ & 1.05 \\
\hline $\mathrm{L}_{2} \mathrm{D}_{2}$ & 1.19 \\
\hline $\mathrm{L}_{3} \mathrm{D}_{2}$ & 1.49 \\
\hline S.E N \pm & 0.078 \\
\hline C.D. at $5 \%$ & 0.21 \\
\hline \multicolumn{2}{|c|}{ Interaction $(T \times D)$} \\
\hline $\mathrm{T}_{1} \mathrm{D}_{1}$ & 1.17 \\
\hline $\mathrm{T}_{1} \mathrm{D}_{2}$ & 1.29 \\
\hline $\mathrm{T}_{2} \mathrm{D}_{1}$ & 1.10 \\
\hline $\mathrm{T}_{2} \mathrm{D}_{2}$ & 1.15 \\
\hline $\mathrm{T}_{3} \mathrm{D}_{1}$ & 0.95 \\
\hline $\mathrm{T}_{3} \mathrm{D}_{2}$ & 1.10 \\
\hline S.E N \pm & 0.078 \\
\hline C.D. at $5 \%$ & 0.21 \\
\hline
\end{tabular}




\begin{tabular}{|c|c|c|}
\hline \multicolumn{2}{|c|}{ Interaction $(\mathrm{L} \times \mathrm{T})$} & 90DAP \\
\hline \multicolumn{2}{|c|}{$\mathrm{T}_{1} \mathrm{~L}_{1}$} & 1.08 \\
\hline \multicolumn{2}{|l|}{$\mathrm{T}_{1} \mathrm{~L}_{2}$} & 1.20 \\
\hline \multicolumn{2}{|l|}{$\mathrm{T}_{1} \mathrm{~L}_{3}$} & 1.42 \\
\hline \multicolumn{2}{|l|}{$\mathrm{T}_{2} \mathrm{~L}_{1}$} & 1.11 \\
\hline \multicolumn{2}{|l|}{$\mathrm{T}_{2} \mathrm{~L}_{2}$} & 1.01 \\
\hline \multicolumn{2}{|l|}{$\mathrm{T}_{2} \mathrm{~L}_{3}$} & 1.20 \\
\hline \multicolumn{2}{|l|}{$\mathrm{T}_{3} \mathrm{~L}_{1}$} & 0.85 \\
\hline \multicolumn{2}{|l|}{$\mathrm{T}_{3} \mathrm{~L}_{2}$} & 1.06 \\
\hline \multicolumn{2}{|l|}{$\mathrm{T}_{3} \mathrm{~L}_{3}$} & 1.17 \\
\hline \multicolumn{2}{|l|}{ S.E N \pm} & 0.096 \\
\hline \multicolumn{2}{|l|}{ C.D. at $5 \%$} & 0.26 \\
\hline \multicolumn{3}{|c|}{ Interaction $(T \times \mathrm{D} \times \mathrm{L})$} \\
\hline \multicolumn{2}{|l|}{$\mathrm{T}_{1} \mathrm{D}_{1} \mathrm{~L}_{1}$} & 0.86 \\
\hline \multicolumn{2}{|l|}{$\mathrm{T}_{1} \mathrm{D}_{1} \mathrm{~L}_{2}$} & 1.12 \\
\hline \multicolumn{2}{|l|}{$\mathrm{T}_{1} \mathrm{D}_{1} \mathrm{~L}_{3}$} & 1.24 \\
\hline \multicolumn{2}{|l|}{$\mathrm{T}_{1} \mathrm{D}_{2} \mathrm{~L}_{1}$} & 1.31 \\
\hline \multicolumn{2}{|l|}{$\mathrm{T}_{1} \mathrm{D}_{2} \mathrm{~L}_{2}$} & 1.27 \\
\hline \multicolumn{2}{|l|}{$\mathrm{T}_{1} \mathrm{D}_{2} \mathrm{~L}_{3}$} & 1.45 \\
\hline \multicolumn{2}{|l|}{$\mathrm{T}_{2} \mathrm{D}_{1} \mathrm{~L}_{1}$} & 1.27 \\
\hline \multicolumn{2}{|l|}{$\mathrm{T}_{2} \mathrm{D}_{1} \mathrm{~L}_{2}$} & 1.20 \\
\hline \multicolumn{2}{|l|}{$\mathrm{T}_{2} \mathrm{D}_{1} \mathrm{~L}_{3}$} & 1.13 \\
\hline \multicolumn{2}{|l|}{$\mathrm{T}_{2} \mathrm{D}_{2} \mathrm{~L}_{1}$} & 0.95 \\
\hline \multicolumn{2}{|l|}{$\mathrm{T}_{2} \mathrm{D}_{2} \mathrm{~L}_{2}$} & 1.01 \\
\hline \multicolumn{2}{|l|}{$\mathrm{T}_{2} \mathrm{D}_{2} \mathrm{~L}_{3}$} & 1.15 \\
\hline \multicolumn{2}{|l|}{$\mathrm{T}_{3} \mathrm{D}_{1} \mathrm{~L}_{1}$} & 0.82 \\
\hline \multicolumn{2}{|l|}{$\mathrm{T}_{3} \mathrm{D}_{1} \mathrm{~L}_{2}$} & 1.02 \\
\hline \multicolumn{2}{|l|}{$\mathrm{T}_{3} \mathrm{D}_{1} \mathrm{~L}_{3}$} & 1.05 \\
\hline \multicolumn{2}{|l|}{$\mathrm{T}_{3} \mathrm{D}_{2} \mathrm{~L}_{1}$} & 0.89 \\
\hline $\mathrm{T}_{3} \mathrm{D}_{2} \mathrm{~L}_{2}$ & & 1.10 \\
\hline $\mathrm{T}_{3} \mathrm{D}_{2} \mathrm{~L}_{3}$ & & 1.33 \\
\hline S.E N \pm & & 0.13 \\
\hline $\mathrm{CD}$ at $5 \%$ & & 0.37 \\
\hline & Treatment details & \\
\hline $\mathrm{D}_{1}$-Diameter $1 \mathrm{~cm}$ & $\mathrm{~L}_{1}$ - Length $10 \mathrm{~cm}$ & $\mathrm{~T}_{1}-3000 \mathrm{ppm} \mathrm{IBA}$ \\
\hline & $\mathrm{L}_{2}$ - Length $15 \mathrm{~cm}$ & $\mathrm{~T}_{2^{-}} 4000 \mathrm{ppm}$ IBA \\
\hline $\mathrm{D}_{2}$-Dimeter $1.5 \mathrm{~cm}$ & $\mathrm{~L}_{3}$ - Length $20 \mathrm{~cm}$ & $\mathrm{~T}_{3}-5000 \mathrm{ppm} \mathrm{IBA}$ \\
\hline
\end{tabular}


Table.8 Effect of cutting size and IBA concentration on number of leaves per cutting in pomegranate

\begin{tabular}{|c|c|c|c|}
\hline \multirow[t]{2}{*}{ Treatments } & \multicolumn{3}{|c|}{ Number of leaves per cutting } \\
\hline & 30DAP & 60DAP & 90DAP \\
\hline \multicolumn{4}{|c|}{$\begin{array}{l}\text { Diameter of cutting } \\
\text { (D) }\end{array}$} \\
\hline$D_{1}$ & 21.34 & 36.41 & 48.49 \\
\hline $\mathrm{D}_{2}$ & 23.85 & 37.87 & 50.38 \\
\hline S.E N \pm & 0.74 & 1.11 & 1.64 \\
\hline C.D. at $5 \%$ & 2.06 & NS & NS \\
\hline \multicolumn{4}{|c|}{ Length of cutting (L) } \\
\hline $\mathrm{L}_{1}$ & 21.42 & 34.12 & 49.08 \\
\hline $\mathrm{L}_{2}$ & 20.35 & 36.53 & 48.65 \\
\hline $\mathrm{L}_{3}$ & 26.02 & 40.77 & 50.57 \\
\hline S.E N \pm & 0.91 & 1.36 & 2.01 \\
\hline C.D. at $5 \%$ & 2.53 & 3.77 & NS \\
\hline \multicolumn{4}{|c|}{ IBA concentration $(\mathrm{T})$} \\
\hline $\mathrm{T}_{1}$ & 24.64 & 42.50 & 59.25 \\
\hline $\mathrm{T}_{2}$ & 19.31 & 34.25 & 45.16 \\
\hline $\mathrm{T}_{3}$ & 21.84 & 37.67 & 50.78 \\
\hline S.E N \pm & 0.91 & 1.36 & 2.01 \\
\hline C.D. at $5 \%$ & 2.53 & 3.77 & 5.57 \\
\hline \multicolumn{4}{|c|}{ Interaction $(\mathrm{L} \times \mathrm{D})$} \\
\hline $\mathrm{L}_{1} \mathrm{D}_{1}$ & 20.51 & 35.17 & 50.51 \\
\hline $\mathrm{L}_{2} \mathrm{D}_{1}$ & 19.46 & 36.04 & 41.66 \\
\hline $\mathrm{L}_{3} \mathrm{D}_{1}$ & 23.04 & 38.02 & 49.31 \\
\hline$L_{1} D_{2}$ & 22.33 & 33.06 & 47.66 \\
\hline $\mathrm{L}_{2} \mathrm{D}_{2}$ & 20.24 & 37.02 & 44.64 \\
\hline $\mathrm{L}_{3} \mathrm{D}_{2}$ & 29.00 & 43.53 & 58.84 \\
\hline S.E N \pm & 1.29 & 1.93 & 1.25 \\
\hline C.D. at $5 \%$ & 3.58 & 5.34 & 3.75 \\
\hline \multicolumn{4}{|c|}{ Interaction ( $\mathrm{T} \times \mathrm{D})$} \\
\hline $\mathrm{T}_{1} \mathrm{D}_{1}$ & 25.11 & 38.64 & 46.95 \\
\hline $\mathrm{T}_{1} \mathrm{D}_{2}$ & 29.17 & 43.35 & 57.77 \\
\hline $\mathrm{T}_{2} \mathrm{D}_{1}$ & 22.33 & 34.93 & 43.91 \\
\hline $\mathrm{T}_{2} \mathrm{D}_{2}$ & 23.28 & 35.57 & 48.42 \\
\hline $\mathrm{T}_{3} \mathrm{D}_{1}$ & 19.57 & 32.66 & 41.62 \\
\hline $\mathrm{T}_{3} \mathrm{D}_{2}$ & 24.11 & 37.68 & 45.95 \\
\hline S.E N \pm & 1.29 & 1.93 & 1.84 \\
\hline C.D. at $5 \%$ & 3.58 & 5.34 & 5.45 \\
\hline
\end{tabular}




\begin{tabular}{|c|c|c|c|}
\hline Interaction $(\mathrm{L} \times \mathrm{T})$ & 30DAP & 60DAP & 90DAP \\
\hline $\mathrm{T}_{1} \mathrm{~L}_{1}$ & 25.60 & 36.93 & 49.26 \\
\hline $\mathrm{T}_{1} \mathrm{~L}_{2}$ & 27.60 & 38.36 & 47.70 \\
\hline $\mathrm{T}_{1} \mathrm{~L}_{3}$ & 30.73 & 49.20 & 60.13 \\
\hline $\mathrm{T}_{2} \mathrm{~L}_{1}$ & 22.06 & 33.16 & 51.56 \\
\hline $\mathrm{T}_{2} \mathrm{~L}_{2}$ & 19.23 & 34.20 & 46.96 \\
\hline $\mathrm{T}_{2} \mathrm{~L}_{3}$ & 25.63 & 38.40 & 44.96 \\
\hline $\mathrm{T}_{3} \mathrm{~L}_{1}$ & 23.60 & 36.26 & 49.43 \\
\hline $\mathrm{T}_{3} \mathrm{~L}_{2}$ & 24.23 & 37.03 & 50.30 \\
\hline $\mathrm{T}_{3} \mathrm{~L}_{3}$ & 26.70 & 40.73 & 44.63 \\
\hline S.E N \pm & 1.58 & 2.36 & 3.48 \\
\hline C.D. at $5 \%$ & 4.38 & 6.54 & 9.65 \\
\hline \multicolumn{4}{|l|}{ Interaction $(\mathrm{T} \times \mathrm{D} \times \mathrm{L})$} \\
\hline $\mathrm{T}_{1} \mathrm{D}_{1} \mathrm{~L}_{1}$ & 23.20 & 35.46 & 44.60 \\
\hline $\mathrm{T}_{1} \mathrm{D}_{1} \mathrm{~L}_{2}$ & 24.30 & 40.40 & 46.33 \\
\hline $\mathrm{T}_{1} \mathrm{D}_{1} \mathrm{~L}_{3}$ & 26.51 & 40.06 & 55.93 \\
\hline $\mathrm{T}_{1} \mathrm{D}_{2} \mathrm{~L}_{1}$ & 23.00 & 38.40 & 49.93 \\
\hline $\mathrm{T}_{1} \mathrm{D}_{2} \mathrm{~L}_{2}$ & 20.20 & 36.33 & 50.06 \\
\hline $\mathrm{T}_{1} \mathrm{D}_{2} \mathrm{~L}_{3}$ & 36.33 & 48.33 & 68.33 \\
\hline $\mathrm{T}_{2} \mathrm{D}_{1} \mathrm{~L}_{1}$ & 22.46 & 35.00 & 52.46 \\
\hline $\mathrm{T}_{2} \mathrm{D}_{1} \mathrm{~L}_{2}$ & 18.26 & 33.40 & 41.93 \\
\hline $\mathrm{T}_{2} \mathrm{D}_{1} \mathrm{~L}_{3}$ & 23.26 & 37.40 & 46.33 \\
\hline $\mathrm{T}_{2} \mathrm{D}_{2} \mathrm{~L}_{1}$ & 21.66 & 31.33 & 47.66 \\
\hline $\mathrm{T}_{2} \mathrm{D}_{2} \mathrm{~L}_{2}$ & 24.20 & 36.00 & 54.00 \\
\hline $\mathrm{T}_{2} \mathrm{D}_{2} \mathrm{~L}_{3}$ & 27.00 & 39.40 & 43.60 \\
\hline $\mathrm{T}_{3} \mathrm{D}_{1} \mathrm{~L}_{1}$ & 19.86 & 38.06 & 51.46 \\
\hline $\mathrm{T}_{3} \mathrm{D}_{1} \mathrm{~L}_{2}$ & 20.13 & 35.33 & 48.73 \\
\hline $\mathrm{T}_{3} \mathrm{D}_{1} \mathrm{~L}_{3}$ & 23.73 & 36.60 & 46.66 \\
\hline $\mathrm{T}_{3} \mathrm{D}_{2} \mathrm{~L}_{1}$ & 23.33 & 37.46 & 45.40 \\
\hline $\mathrm{T}_{3} \mathrm{D}_{2} \mathrm{~L}_{2}$ & 25.33 & 38.73 & 51.86 \\
\hline $\mathrm{T}_{3} \mathrm{D}_{2} \mathrm{~L}_{3}$ & 28.66 & 42.86 & 43.60 \\
\hline S.E N \pm & 2.24 & 2.34 & 4.93 \\
\hline $\mathrm{CD}$ at $5 \%$ & 6.20 & 6.25 & 13.65 \\
\hline & reatment & & \\
\hline $\mathrm{D}_{1}$-Diameter $1 \mathrm{~cm}$ & $\mathrm{~L}_{1}$ - Lengtl & & ppm IBA \\
\hline & $\mathrm{L}_{2}$ - Lengtl & & ppm IBA \\
\hline $\mathrm{D}_{2}$-Dimeter $1.5 \mathrm{~cm}$ & $\mathrm{~L}_{3}$ - Lengtl & & ppm IBA \\
\hline
\end{tabular}


Table.9 Effect of cutting size and IBA concentration on leaf area of pomegranate cuttings

\begin{tabular}{|c|c|}
\hline \multirow[t]{2}{*}{ Treatments } & Leaf area $\left(\mathrm{cm}^{2}\right)$ \\
\hline & 90DAP \\
\hline \multicolumn{2}{|c|}{ Diameter of cutting (D) } \\
\hline$D_{1}$ & 31.57 \\
\hline $\mathrm{D}_{2}$ & 36.86 \\
\hline S.E N \pm & 0.19 \\
\hline C.D. at $5 \%$ & 0.53 \\
\hline \multicolumn{2}{|c|}{ Length of cutting (L) } \\
\hline $\mathrm{L}_{1}$ & 34.04 \\
\hline $\mathrm{L}_{2}$ & 36.09 \\
\hline $\mathrm{L}_{3}$ & 38.36 \\
\hline S.E N \pm & 0.23 \\
\hline C.D. at $5 \%$ & 0.65 \\
\hline \multicolumn{2}{|c|}{ IBA concentration $(\mathrm{T})$} \\
\hline $\mathrm{T}_{1}$ & 36.06 \\
\hline $\mathrm{T}_{2}$ & 35.64 \\
\hline $\mathrm{T}_{3}$ & 34.15 \\
\hline S.E N \pm & 0.23 \\
\hline C.D. at $5 \%$ & 0.65 \\
\hline \multicolumn{2}{|c|}{ Interaction $(\mathrm{L} \times \mathrm{D})$} \\
\hline $\mathrm{L}_{1} \mathrm{D}_{1}$ & 34.06 \\
\hline $\mathrm{L}_{2} \mathrm{D}_{1}$ & 35.43 \\
\hline $\mathrm{L}_{3} \mathrm{D}_{1}$ & 36.93 \\
\hline $\mathrm{L}_{1} \mathrm{D}_{2}$ & 34.02 \\
\hline $\mathrm{L}_{2} \mathrm{D}_{2}$ & 36.75 \\
\hline $\mathrm{L}_{3} \mathrm{D}_{2}$ & 39.80 \\
\hline S.E N \pm & 0.33 \\
\hline C.D. at $5 \%$ & 0.92 \\
\hline \multicolumn{2}{|c|}{ Interaction $(\mathrm{T} \times \mathrm{D})$} \\
\hline $\mathrm{T}_{1} \mathrm{D}_{1}$ & 35.36 \\
\hline$T_{1} D_{2}$ & 36.76 \\
\hline $\mathrm{T}_{2} \mathrm{D}_{1}$ & 35.66 \\
\hline $\mathrm{T}_{2} \mathrm{D}_{2}$ & 38.69 \\
\hline $\mathrm{T}_{3} \mathrm{D}_{1}$ & 33.40 \\
\hline $\mathrm{T}_{3} \mathrm{D}_{2}$ & 35.12 \\
\hline S.E N \pm & 0.33 \\
\hline C.D. at $5 \%$ & 0.92 \\
\hline
\end{tabular}




\begin{tabular}{|c|c|c|}
\hline \multicolumn{2}{|c|}{ Interaction $(\mathrm{L} \times \mathrm{T})$} & 90DAP \\
\hline \multicolumn{2}{|l|}{$\mathrm{T}_{1} \mathrm{~L}_{1}$} & 33.06 \\
\hline \multicolumn{2}{|l|}{$\mathrm{T}_{1} \mathrm{~L}_{2}$} & 36.14 \\
\hline \multicolumn{2}{|l|}{$\mathrm{T}_{1} \mathrm{~L}_{3}$} & 38.98 \\
\hline \multicolumn{2}{|l|}{$\mathrm{T}_{2} \mathrm{~L}_{1}$} & 36.56 \\
\hline \multicolumn{2}{|l|}{$\mathrm{T}_{2} \mathrm{~L}_{2}$} & 35.99 \\
\hline \multicolumn{2}{|l|}{$\mathrm{T}_{2} \mathrm{~L}_{3}$} & 37.48 \\
\hline \multicolumn{2}{|l|}{$\mathrm{T}_{3} \mathrm{~L}_{1}$} & 30.51 \\
\hline \multicolumn{2}{|l|}{$\mathrm{T}_{3} \mathrm{~L}_{2}$} & 33.14 \\
\hline \multicolumn{2}{|l|}{$\mathrm{T}_{3} \mathrm{~L}_{3}$} & 35.64 \\
\hline \multicolumn{2}{|l|}{ S.E N土 } & 0.40 \\
\hline \multicolumn{2}{|l|}{ C.D. at $5 \%$} & 0.11 \\
\hline \multicolumn{3}{|c|}{ Interaction $(\mathrm{T} \times \mathrm{D} \times \mathrm{L})$} \\
\hline \multicolumn{2}{|l|}{$\mathrm{T}_{1} \mathrm{D}_{1} \mathrm{~L}_{1}$} & 34.04 \\
\hline \multicolumn{2}{|l|}{$\mathrm{T}_{1} \mathrm{D}_{1} \mathrm{~L}_{2}$} & 35.14 \\
\hline \multicolumn{2}{|l|}{$\mathrm{T}_{1} \mathrm{D}_{1} \mathrm{~L}_{3}$} & 36.88 \\
\hline \multicolumn{2}{|l|}{$\mathrm{T}_{1} \mathrm{D}_{2} \mathrm{~L}_{1}$} & 32.07 \\
\hline \multicolumn{2}{|l|}{$\mathrm{T}_{1} \mathrm{D}_{2} \mathrm{~L}_{2}$} & 37.13 \\
\hline \multicolumn{2}{|l|}{$\mathrm{T}_{1} \mathrm{D}_{2} \mathrm{~L}_{3}$} & 41.07 \\
\hline \multicolumn{2}{|l|}{$\mathrm{T}_{2} \mathrm{D}_{1} \mathrm{~L}_{1}$} & 33.11 \\
\hline \multicolumn{2}{|l|}{$\mathrm{T}_{2} \mathrm{D}_{1} \mathrm{~L}_{2}$} & 34.99 \\
\hline \multicolumn{2}{|l|}{$\mathrm{T}_{2} \mathrm{D}_{1} \mathrm{~L}_{3}$} & 35.88 \\
\hline \multicolumn{2}{|l|}{$\mathrm{T}_{2} \mathrm{D}_{2} \mathrm{~L}_{1}$} & 34.00 \\
\hline \multicolumn{2}{|l|}{$\mathrm{T}_{2} \mathrm{D}_{2} \mathrm{~L}_{2}$} & 36.99 \\
\hline \multicolumn{2}{|l|}{$\mathrm{T}_{2} \mathrm{D}_{2} \mathrm{~L}_{3}$} & 38.07 \\
\hline \multicolumn{2}{|l|}{$\mathrm{T}_{3} \mathrm{D}_{1} \mathrm{~L}_{1}$} & 30.00 \\
\hline \multicolumn{2}{|l|}{$\mathrm{T}_{3} \mathrm{D}_{1} \mathrm{~L}_{2}$} & 33.14 \\
\hline \multicolumn{2}{|l|}{$\mathrm{T}_{3} \mathrm{D}_{1} \mathrm{~L}_{3}$} & 35.03 \\
\hline $\mathrm{T}_{3} \mathrm{D}_{2} \mathrm{~L}_{1}$ & & 30.99 \\
\hline $\mathrm{T}_{3} \mathrm{D}_{2} \mathrm{~L}_{2}$ & & 35.13 \\
\hline $\mathrm{T}_{3} \mathrm{D}_{2} \mathrm{~L}_{3}$ & & 37.24 \\
\hline S.E N \pm & & 0.57 \\
\hline $\mathrm{CD}$ at $5 \%$ & & 0.15 \\
\hline & Treatment details & \\
\hline $\mathrm{D}_{1}$-Diameter $1 \mathrm{~cm}$ & $\mathrm{~L}_{1}$ - Length $10 \mathrm{~cm}$ & $\mathrm{~T}_{1^{-}} 3000 \mathrm{ppm} \mathrm{IBA}$ \\
\hline & $\mathrm{L}_{2}$ - Length $15 \mathrm{~cm}$ & $\mathrm{~T}_{2^{-}} 4000 \mathrm{ppm} \mathrm{IBA}$ \\
\hline $\mathrm{D}_{2}$-Dimeter $1.5 \mathrm{~cm}$ & $\mathrm{~L}_{3}$ - Length $20 \mathrm{~cm}$ & $\mathrm{~T}_{3^{-}} 5000 \mathrm{ppm} \mathrm{IBA}$ \\
\hline
\end{tabular}

\section{Interaction effect of $(\mathrm{L} \times \mathrm{D})$}

Significantly maximum number of branches (4.22) was observed in interaction of $\mathrm{L}_{3} \mathrm{D}_{2}$ i.e. (length $20 \mathrm{~cm}$ and $1.5 \mathrm{~cm}$ diameter), and minimum number of branches (2.55) was observed in $\mathrm{L}_{1} \mathrm{D}_{2}$ i.e. (length $10 \mathrm{~cm}$ and 1.5 cm diameter).

\section{Interaction effect of $(\mathrm{T} \times \mathrm{D})$}

Significantly maximum number of branches per cutting (4.06) was observed in interaction of $\mathrm{T}_{1} \mathrm{D}_{2}$ i.e. (IBA $3000 \mathrm{ppm}$ and $1.5 \mathrm{~cm}$ diameter) and minimum number of branches (3.00) was observed in interaction of $\mathrm{T}_{3} \mathrm{D}_{1}$.

\section{Interaction effect of $(T \times L)$}

The interaction effect of length of cutting and IBA concentration on number of branches was significant.

The interaction of $\mathrm{T}_{1} \mathrm{~L}_{3}$ i.e. (IBA $3000 \mathrm{ppm}$ and $20 \mathrm{~cm}$ length) was recorded significantly maximum number of branches (4.66) which was statistically at par with interactions of $\mathrm{T}_{2} \mathrm{~L}_{3}$ (4.16). However, minimum number of 
branches per cutting (2.30) was observed in $\mathrm{T}_{3} \mathrm{~L}_{1}$. i.e. (IBA $5000 \mathrm{ppm}$ and $10 \mathrm{~cm}$ length).

\section{Interaction effect of $(T \times D \times L)$}

The interaction of $\mathrm{T}_{1} \mathrm{D}_{2} \mathrm{~L}_{3}$ recorded significantly maximum number of branches (5.13) which was statistically at par with interactions of $\mathrm{T}_{2} \mathrm{D}_{2} \mathrm{~L}_{3}(4.33)$

whereas minimum number of branches per cuttings (2.13) was observed in $\mathrm{T}_{3} \mathrm{D}_{2} \mathrm{~L}_{1}$.

\section{Number of branches per cutting at 90DAP}

Data regarding number of branches per cuttings at survival stage (90 DAP) as influenced by diameter of cutting (D), length of cutting (L), IBA concentrations (T) and their interactions are presented in Table 3.

\section{Effect of cutting diameter (D)}

The data do not show significant effect of cutting diameter on number of branches per cutting.

\section{Effect of cutting length ( $L)$}

The perusal of data in Table 4 reveals that, significantly maximum number of branches per cutting (4.22) was recorded in $\mathrm{L}_{3}$ i.e. (20 $\mathrm{cm}$ length) and minimum (3.17) were observed in $\mathrm{L}_{1}$ i.e. (10 cm length) at 90 DAP. This might be due to highest branches at 30 DAP and 60 DAP. Significantly highest leaf area was available for photosynthesis and food material which helps to increase number of branches at survival. Similar results were also reported by Jadhav et al., (2003) and Devi et al., (2016).

\section{IBA concentration $(T)$}

Significantly maximum number of branches for pomegranate cuttings (4.12) at 90 DAP was observed in $\mathrm{T}_{1}$ i.e. (IBA $3000 \mathrm{ppm}$ ) and minimum number of branches (3.15) were observed in of $\mathrm{T}_{3}$ i.e. (IBA $5000 \mathrm{ppm}$ ). This might be due to vigorous root system which increased nutrient uptake under the combined influence of IBA and PHB application.

It affected the cell division in the vascular cambium, cell expansion and control of differentiation into different types of cambial resulting in increase in number of shoots. Devi et al., (2016)

\section{Interaction effect of $(\mathbf{L} \times \mathbf{D})$}

Significantly maximum number of branches per cutting (4.53) was observed in interaction of $\mathrm{L}_{3} \mathrm{D}_{2}$ i.e. (length $20 \mathrm{~cm}$ and $1.5 \mathrm{~cm}$ diameter), however minimum number of branches (3.08) was observed in interaction of $\mathrm{L}_{1} \mathrm{D}_{2}$ i.e. (length $10 \mathrm{~cm}$ and $1.5 \mathrm{~cm}$ diameter) at 90 DAP.

\section{Interaction effect of $(\mathbf{T} \times \mathbf{D})$}

Significantly maximum number of branches per cutting (4.48) was observed in interaction of $\mathrm{T}_{1} \mathrm{D}_{2}$ i.e. (IBA $3000 \mathrm{ppm}$ and $1.5 \mathrm{~cm}$ diameter) and minimum number of branches (3.11) was observed in interaction of $\mathrm{T}_{3} \mathrm{D}_{1}$ i.e. (IBA $5000 \mathrm{ppm}$ and $1 \mathrm{~cm}$ diameter) at 90 DAP.

\section{Interaction effect of $(T \times L)$}

The interaction effect of length of cutting and IBA concentration on number of branches shows significant variation.

The interaction of $\mathrm{T}_{1} \mathrm{~L}_{3}$ i.e. (IBA 3000 ppm and $20 \mathrm{~cm}$ length) was recorded significantly highest number of branches (5.16) per cutting which was statistically at par with interaction of $\mathrm{T}_{2} \mathrm{~L}_{3}$ (4.03), while lowest number of branches (2.83) was observed in interaction of $\mathrm{T}_{2} \mathrm{~L}_{1}$ i.e. (IBA $4000 \mathrm{ppm}$ and $1 \mathrm{~cm}$ diameter). 
However, interaction of IBA, diameter and length of cuttings $(T \times D \times L)$ on number of branches per cutting was found to be non significant.

\section{Shoot length (cm) at 60 DAP}

It is evident from the data that shoot length at 60 DAP of pomegranate cuttings as influenced by diameter of cutting (D), length of cutting (L), IBA concentrations (T) and their interaction are presented in Table 4, effect of cutting diameter on shoot length was found to be non significant.

\section{Effect of cutting length $(\mathrm{L})$}

The data presented in Table 4 reveals that, significantly highest shoot length $(15.08 \mathrm{~cm})$ was recorded in $\mathrm{L}_{3}$ i.e. (20 cm length) and lowest $(12.91 \mathrm{~cm})$ was observed in $\mathrm{L}_{2}$ i.e. $(15$ cm length).

This might be due to better physiological maturity and higher amount of reserve food materials in the cuttings. Pooja et al., (2013).

\section{IBA concentration $(T)$}

Significantly maximum shoot length (15.38 $\mathrm{cm}$ ) was observed in treatment $\mathrm{T}_{1}$ i.e. (IBA $3000 \mathrm{ppm})$ and minimum shoot length (12.86 $\mathrm{cm}$ ) was recorded in treatment $\mathrm{T}_{3}$ i.e. (IBA $5000 \mathrm{ppm}$ ). This might be due to higher cell activity, more synthesized food material and photosynthates (Kamboj, 2017).

\section{Interaction effect of $(\mathrm{L} \times \mathrm{D})$}

According to data regarding shoot length significant variation was observed, maximum shoot length $(15.77 \mathrm{~cm})$ was recorded in interaction of $\mathrm{L}_{3} \mathrm{D}_{2}$ i.e. (length $15 \mathrm{~cm}$ and 1.5 $\mathrm{cm}$ diameter) while minimum shoot length $(12.35 \mathrm{~cm})$ was observed in interaction of $\mathrm{L}_{2} \mathrm{D}_{1}$ i.e. (length $15 \mathrm{~cm}$ and $1 \mathrm{~cm}$ diameter).

\section{Interaction effect of $(\mathbf{T} \times \mathbf{D})$}

Significantly highest shoot length $(16.86 \mathrm{~cm})$ was recorded with interaction of $\mathrm{T}_{1} \mathrm{D}_{2}$ i.e. (IBA $3000 \mathrm{ppm}$ and $1.5 \mathrm{~cm}$ diameter) and lowest shoot length $(12.57 \mathrm{~cm})$ was measured in treatment combination of $\mathrm{T}_{3} \mathrm{D}_{1}$ i.e. (IBA $5000 \mathrm{ppm}$ and $1 \mathrm{~cm}$ diameter).

\section{Interaction effect of $(\mathrm{T} \times \mathrm{L})$}

The interaction effect of length of cutting and IBA concentration on shoot length shows significant variation. The interaction of $\mathrm{T}_{1} \mathrm{~L}_{3}$ i.e. (IBA $3000 \mathrm{ppm}$ and $20 \mathrm{~cm}$ length) was recorded significantly maximum shoot length $(16.23 \mathrm{~cm})$ and minimum shoot length $(11.23$ $\mathrm{cm}$ ) was observed in interaction of $\mathrm{T}_{3} \mathrm{~L}_{2}$.

\section{Interaction effect of $(T \times D \times L)$}

The interaction of IBA concentrations, diameter and length of cuttings on shoot length was found significant. The interaction of $\mathrm{T}_{1} \mathrm{D}_{2} \mathrm{~L}_{3}$ recorded significantly maximum shoot length $(18.80 \mathrm{~cm})$, which was followed by interactions of $\mathrm{T}_{1} \mathrm{D}_{2} \mathrm{~L}_{2}(16.06 \mathrm{~cm})$ and $\mathrm{T}_{1} \mathrm{D}_{2} \mathrm{~L}_{1}(15.73 \mathrm{~cm})$. Contrary to this, minimum shoot length $(11.20 \mathrm{~cm})$ was measured under interaction of $\mathrm{T}_{3} \mathrm{D}_{1} \mathrm{~L}_{2}$.

\section{Shoot length (cm) at 90DAP}

The effect of diameter of cutting (D), length of cutting (L), IBA concentrations (T) and their interaction on shoot length at survival (90DAP) of pomegranate cuttings are presented in Table 4, effect of cutting diameter on shoot length was statistically non significant.

\section{Effect of cutting length ( $L)$}

Significantly maximum shoot length $(18.26 \mathrm{~cm})$ was recorded in treatment $\mathrm{L}_{3}$ i.e. (20 cm length) and minimum shoot length 
$(16.54 \mathrm{~cm})$ was observed in $\mathrm{L}_{1}$ i.e. $(10 \mathrm{~cm}$ length). This might be due to better physiological maturity and higher amount of reserve food materials in the cuttings (Pooja et al., 2013).

\section{IBA concentration $(T)$}

Maximum shoot length $(18.25 \mathrm{~cm})$ per cutting was observed in treatment $\mathrm{T}_{1}$ i.e. application of IBA $3000 \mathrm{ppm}$ which was followed by $\mathrm{T}_{2}$ $(17.71 \mathrm{~cm})$, and minimum shoot length $(16.48$ $\mathrm{cm})$ was recorded in $\mathrm{T}_{3}$ i.e. IBA $3000 \mathrm{ppm}$. This might be due to higher cell activity, more synthesized food material and photosynthates (Kamboj, 2017).

\section{Interaction effect of $(\mathrm{L} \times \mathrm{D})$}

According to analysed of data regarding shoot length, significant variation was observed in interaction between the length and diameter of cutting. Significantly maximum shoot length $(19.17 \mathrm{~cm})$ was recorded in interaction of $\mathrm{L}_{3} \mathrm{D}_{2}$ i.e. (length $20 \mathrm{~cm}$ and $1.5 \mathrm{~cm}$ diameter) and minimum shoot length $(16.28 \mathrm{~cm})$ was observed in interaction of $\mathrm{L}_{1} \mathrm{D}_{1}$ i.e. (length 10 $\mathrm{cm}$ and $1 \mathrm{~cm}$ diameter).

\section{Interaction effect of $(T \times D)$}

Significantly maximum shoot length (19.40 $\mathrm{cm})$ was recorded with interaction of $\mathrm{T}_{1} \mathrm{D}_{2}$ i.e. (IBA $3000 \mathrm{ppm}$ and $1.5 \mathrm{~cm}$ diameter) which was followed by $T_{2} D_{1}(18.33 \mathrm{~cm})$ and $T_{1} D_{1}$ $(17.11 \mathrm{~cm})$, and minimum shoot length $(15.97$ $\mathrm{cm}$ ) was observed in treatment combination of $\mathrm{T}_{3} \mathrm{D}_{1}$ i.e. (IBA $5000 \mathrm{ppm}$ and $1.5 \mathrm{~cm}$ diameter).

\section{Interaction effect of $(T \times L)$}

The interaction effect of length of cutting and IBA concentration on shoot length shows significant variation. The interaction $\mathrm{T}_{1} \mathrm{~L}_{3}$ i.e. (IBA $3000 \mathrm{ppm}$ and $20 \mathrm{~cm}$ length) was recorded significantly maximum shoot length $(20.20 \mathrm{~cm})$ which was statistically at par with interactions of $\mathrm{T}_{1} \mathrm{~L}_{2}(18.70 \mathrm{~cm})$ and $\mathrm{T}_{2} \mathrm{~L}_{2}$ $(17.90 \mathrm{~cm})$ however, minimum shoot length $(15.86 \mathrm{~cm})$ was observed in interaction of $\mathrm{T}_{1} \mathrm{~L}_{1}$.

\section{Interaction effect of $(T \times D \times L)$}

The interaction of IBA concentrations, diameter and length of cuttings show significant variation on shoot length of cutting. The interaction of $\mathrm{T}_{1} \mathrm{D}_{2} \mathrm{~L}_{3}$ was recorded maximum shoot length $(21.93 \mathrm{~cm})$ which was followed by the interactions of $\mathrm{T}_{1} \mathrm{D}_{1} \mathrm{~L}_{2}(19.06 \mathrm{~cm})$ and $\mathrm{T}_{2} \mathrm{D}_{1} \mathrm{~L}_{1}(18.60 \mathrm{~cm})$ whereas minimum shoot length $(13.80 \mathrm{~cm})$ was measured under interaction of $T_{1} D_{1} L_{1}$.

\section{Shoot diameter (mm) at 60 DAP}

Effect of cutting diameter (D), length of cutting (L), IBA concentrations (T) and their interaction on shoot diameter at $60 \mathrm{DAP}$ of pomegranate cuttings are presented in Table 5, effect of cutting diameter on shoot diameter was found to be non significant.

\section{Effect of cutting length ( $L)$}

Significantly maximum shoot diameter (1.52 $\mathrm{mm})$ was recorded in treatment $\mathrm{L}_{3}$ i.e. $(20 \mathrm{~cm}$ length) and minimum shoot diameter (1.37 $\mathrm{mm}$ ) was observed in $\mathrm{L}_{1}$ i.e. (10 cm length). This might be due to higher cell activity, highest number of branches, leaf area, more synthesized food material and photosynthesis.

Similarly, more shoot diameter attributed to more roots which helps to absorb more nutrients from media and helped in better root and shoot development, there by resulting in better shoots with more shoot diameter. Similar results were also reported by Shukla $e t$ al., (2010), Devi et al., (2016) and Kamboj et al., (2017). 


\section{IBA concentration $(T)$}

Maximum shoot diameter $(1.50 \mathrm{~mm})$ was observed in treatment $\mathrm{T}_{1}$ i.e. (IBA $3000 \mathrm{ppm}$ ) and minimum shoot diameter (1.39) was recorded in treatment $\mathrm{T}_{3}$ i.e. (IBA $5000 \mathrm{ppm}$ ). This might be attributed to more number of roots because auxin favoured cell division and their elongation and helped in better root development there by resulting in better shoots with more shoot diameter (Devi et al., 2016).

\section{Interaction effect of $(\mathrm{L} \times \mathrm{D})$}

According to data regarding shoot diameter, significant variation between the length and diameter was observed. Maximum shoot diameter $(1.56 \mathrm{~mm})$ was recorded in interaction of $\mathrm{L}_{3} \mathrm{D}_{2}$ i.e. (length $20 \mathrm{~cm}$ and 1.5 $\mathrm{cm}$ diameter) and minimum shoot diameter $(1.36 \mathrm{~mm})$ was observed in interaction of $\mathrm{L}_{1} \mathrm{D}_{1}$ i.e. (length $10 \mathrm{~cm}$ and $1 \mathrm{~cm}$ diameter).

\section{Interaction effect of $(T \times L)$}

The interaction effect of length of cutting and IBA concentration on shoot diameter shows significant differences among the interactions. The interaction of $\mathrm{T}_{1} \mathrm{~L}_{3}$ i.e. (IBA $3000 \mathrm{ppm}$ and $20 \mathrm{~cm}$ length) was recorded significantly highest shoot diameter $(1.62 \mathrm{~mm})$, while lowest shoot diameter $(1.40 \mathrm{~mm})$ was observed in interaction of $T_{2} L_{3}$. However, the interaction effect of IBA concentrations, diameter and length of cuttings $(\mathrm{T} \times \mathrm{D} \times \mathrm{L})$ do not show significant differences with respect to number of branches per cutting.

\section{Shoot diameter $(\mathrm{mm})$ at 90DAP}

The shoot diameter at survival (90DAP) of pomegranate cuttings was influenced by diameter of cutting (D), length of cutting (L), IBA concentrations (T) and their interactions are presented in Table 5. Effect of cutting diameter (D), length (L), IBA concentration
(T) and interaction of $(\mathrm{L} \times \mathrm{D})$ on shoot diameter at 90 DAP was found to be non significant.

\section{Interaction effect of $(T \times D)$}

Significantly maximum shoot diameter (1.65 $\mathrm{mm}$ ) was recorded with interaction of $\mathrm{T}_{1} \mathrm{D}_{2}$ i.e. (IBA $3000 \mathrm{ppm}$ and $1.5 \mathrm{~cm}$ diameter) which was followed by $T_{1} D_{1}(1.51 \mathrm{~mm}), T_{2} D_{1}$ $(1.50 \mathrm{~mm})$, and minimum shoot diameter $(1.41$ $\mathrm{mm}$ ) was observed in interaction of $\mathrm{T}_{2} \mathrm{D}_{2}$ at 90 DAP.

\section{Interaction effect of $(T \times L)$}

The interaction effect of length of cutting and IBA concentration on shoot diameter shows significant variation. The interaction $\mathrm{T}_{1} \mathrm{~L}_{3}$ i.e. (IBA $3000 \mathrm{ppm}$ and $20 \mathrm{~cm}$ length) was recorded significantly maximum shoot diameter $(1.74 \mathrm{~mm})$ which was statistically at par with the interactions of $\mathrm{T}_{1} \mathrm{~L}_{2}(1.55 \mathrm{~mm})$, $\mathrm{T}_{2} \mathrm{~L}_{1}(1.52 \mathrm{~mm})$. However, minimum shoot diameter $(1.40 \mathrm{~mm})$ was observed in $\mathrm{T}_{2} \mathrm{~L}_{3}$.

\section{Interaction effect of $(T \times D \times L)$}

The interaction of IBA concentrations, diameter and length of cuttings on shoot diameter was found to be significant. The interaction of $\mathrm{T}_{1} \mathrm{D}_{2} \mathrm{~L}_{3}$ recorded significantly maximum shoot diameter $(1.95 \mathrm{~mm})$ which was followed by $\mathrm{T}_{1} \mathrm{D}_{1} \mathrm{~L}_{2}(1.60 \mathrm{~mm}), \mathrm{T}_{1} \mathrm{D}_{1} \mathrm{~L}_{3}$ $(1.53 \mathrm{~mm})$ and minimum shoot diameter $(1.33$ $\mathrm{mm})$ was measured under interaction of $\mathrm{T}_{2} \mathrm{D}_{2} \mathrm{~L}_{3}$.

The increase in shoot diameter might be due to higher cell activity, more synthesized food material and photosynthesis. Similarly, more shoot diameter at $60 \mathrm{DAP}$ attributed to more roots and helped in better root development there by resulting in better shoots with more shoot diameter. Similar results were also reported by Shukla et al., (2010), Devi et al., (2016) and Kamboj et al., (2017). 


\section{Weight of fresh shoot (g)}

Data regarding weight of fresh shoot of pomegranate cuttings as influenced by diameter of cutting (D), length of cutting (L), IBA concentrations (T) and their interaction are presented in Table 6.

\section{Effect of cutting diameter (D)}

Significantly maximum weight of fresh shoot $(5.84 \mathrm{~g})$ was recorded in $\mathrm{D}_{2}$ i.e. $(1.5 \mathrm{~cm})$ and minimum weight of fresh shoot $(4.54 \mathrm{~g})$ was observed in $\mathrm{D}_{1}$ i.e. $(1 \mathrm{~cm})$. This might be due to early sprouting, maximum growth of shoot which accumulate maximum fresh matter and ultimately maximum weight of fresh shoot. Zhang et al., (2010)

\section{Effect of cutting length $(\mathrm{L})$}

The results clearly indicated that, highest weight of fresh shoot $(6.10 \mathrm{~g})$ was recorded with $\mathrm{L}_{3}$ i.e. $(20 \mathrm{~cm})$ and lowest weight of fresh shoot $(4.02 \mathrm{~g})$ in $\mathrm{L}_{1}$ i.e. (10 $\mathrm{cm}$ length). This might be due to early sprouting and maximum shoots per cutting which play an important role in photosynthesis and hence maximum weight of fresh shoot was recorded by these treatments. Similar results are in accordance with the findings of Stancato et al., (2003), Shukla et al., (2010) and Singh and Singh (2012).

\section{IBA concentration $(T)$}

There was significant variation in the application of IBA concentration. Data regarding weight of fresh shoot per cutting was recorded maximum $(5.91 \mathrm{~g})$ in treatment $\mathrm{T}_{1}$ i.e. (3000 ppm IBA) and minimum (5.51g) was observed in $\mathrm{T}_{2}$ i.e. (IBA $4000 \mathrm{ppm}$ ). Maximum shoot weight might be due to the fact that auxin is for initiation and growth of shoots. This might also be due to the reserved food within the cuttings. Kamboj (2017)

\section{Interaction effect of $(L \times D)$}

The interaction of length and diameter of cutting shows significant effect on weight of fresh shoot. The result came out from data showed that, highest weight of fresh shoot (7.02g) was recorded in interaction of $\mathrm{L}_{3} \mathrm{D}_{2}$ i.e. (length $20 \mathrm{~cm}$ and $1.5 \mathrm{~cm}$ diameter). However, lowest weight of fresh shoot (4.02) was observed in $\mathrm{L}_{1} \mathrm{D}_{1}$ i.e. (length $10 \mathrm{~cm}$ and 1 cm diameter).

\section{Interaction effect of $(T \times D)$}

Significantly highest weight of fresh shoot $(6.88 \mathrm{~g})$ was recorded in interaction of $\mathrm{T}_{1} \mathrm{D}_{2}$ i.e. (IBA $3000 \mathrm{ppm}$ and $1.5 \mathrm{~cm}$ diameter), which was followed by $\mathrm{T}_{3} \mathrm{D}_{2} \quad(6.17 \mathrm{~g})$, $\mathrm{T}_{2} \mathrm{D}_{1}(5.77 \mathrm{~g})$ and lowest weight of fresh shoot $(1.19 \mathrm{~g})$ was observed in interaction of $\mathrm{T}_{3} \mathrm{D}_{1}$. i.e. (IBA 5000 ppm and $1 \mathrm{~cm}$ diameter).

\section{Interaction effect of $(T \times L)$}

The interaction effect of length of cutting and IBA concentration on weight of fresh shoot shows significant variation. The interaction of $\mathrm{T}_{1} \mathrm{~L}_{3}$ i.e. (IBA $3000 \mathrm{ppm}$ and $20 \mathrm{~cm}$ length) was recorded significantly maximum weight of fresh shoot $(6.80 \mathrm{~g})$, which was statistically at par with interactions of $\mathrm{T}_{2} \mathrm{~L}_{3}(6.29 \mathrm{~g})$ and $\mathrm{T}_{3} \mathrm{~L}_{3}$ (6.13g), and minimum weight of fresh shoot $(5.12 \mathrm{~g})$ was observed in $\mathrm{T}_{3} \mathrm{~L}_{1}$. i.e. (IBA $5000 \mathrm{ppm}$ and $10 \mathrm{~cm}$ length). However, weight of fresh shoot was found to be non significant as influenced by IBA concentrations, diameter and length of cutting.

\section{Weight of dry shoot (g)}

Effect of cutting diameter (D), length of cutting (L), IBA concentrations (T) and their interactions on weight of dry shoot presented in Table 6. 


\section{Effect of cutting diameter (D)}

From data in Table 6 it was observed that, maximum weight of dry shoot $(1.74 \mathrm{~g})$ was recorded in $\mathrm{D}_{2}$ i.e. $(1.5 \mathrm{~cm}$ diameter) and minimum weight of dry shoot $(1.22 \mathrm{~g})$ was observed in $\mathrm{D}_{1}$ i.e. $(1 \mathrm{~cm}$ diameter).

This might be due to early sprouting, maximum growth of shoot which accumulate maximum dry matter and ultimately maximum weight of dry shoot. In general cutting diameter, length affect directly on weight of fresh and dry shoot. These results are similar to the findings of Hoad and Leakey (1996), Kaur and Kaur (2016) and Kamboj et al., (2017).

\section{Effect of cutting length ( $L)$}

Significantly maximum weight of dry shoot $(1.99 \mathrm{~g})$ was recorded with $\mathrm{L}_{3}$ i.e. $(20 \mathrm{~cm}$ length) and minimum weight of dry shoot (1.01g) was observed in $\mathrm{L}_{1}$ i.e. (10 cm length).

\section{IBA concentration $(T)$}

Maximum weight of dry shoot (1.76g) was observed in $\mathrm{T}_{1}$ i.e. (IBA $3000 \mathrm{ppm}$ ) and minimum weight of dry shoot $(1.46 \mathrm{~g})$ was observed in $\mathrm{T}_{2}$ i.e. (IBA 4000 ppm). Significantly maximum weight of dry shoots might be due to the fact that auxin is for initiation and growth of shoots. This might also be due to the reserved food within the cuttings (Kamboj, 2017).

\section{Interaction effect of $(\mathrm{L} \times \mathrm{D})$}

The interaction of length and diameter of cutting shows significant variation on weight of dry shoot. Maximum weight of dry shoot (1.96g) was observed in interaction of $\mathrm{L}_{3} \mathrm{D}_{2}$ i.e. (length $20 \mathrm{~cm}$ and $1.5 \mathrm{~cm}$ diameter) and minimum weight of dry shoot $(0.96 \mathrm{~g})$ was observed in interaction of $\mathrm{L}_{1} \mathrm{D}_{1}$ i.e. (length 10 $\mathrm{cm}$ and $1 \mathrm{~cm}$ diameter).

\section{Interaction effect of $(\mathrm{T} \times \mathrm{D})$}

Significantly highest weight of dry shoot $(1.86 \mathrm{~g})$ was observed in interaction of $\mathrm{T}_{1} \mathrm{D}_{2}$ i.e. (IBA $3000 \mathrm{ppm}$ and $1.5 \mathrm{~cm}$ diameter) which was followed by $\mathrm{T}_{3} \mathrm{D}_{2}(1.41 \mathrm{~g})$ and $\mathrm{T}_{2} \mathrm{D}_{2}$ $(1.39 \mathrm{~g})$, and lowest weight of dry shoot $(1.19 \mathrm{~g})$ was observed in interaction of $\mathrm{T}_{3} \mathrm{D}_{1}$ i.e. (IBA $5000 \mathrm{ppm}$ and $1 \mathrm{~cm}$ diameter).

\section{Interaction effect of $(T \times L)$}

The interaction effect of length of cutting and IBA concentration on weight of dry shoot shows significant variation. Interaction of $\mathrm{T}_{1} \mathrm{~L}_{3}$ i.e. (IBA $3000 \mathrm{ppm}$ and $20 \mathrm{~cm}$ length) was recorded significantly maximum weight of dry shoot $(2.42 \mathrm{~g})$, which was statistically at par with interactions of $\mathrm{T}_{2} \mathrm{~L}_{2}(2.12 \mathrm{~g}), \mathrm{T}_{1} \mathrm{~L}_{2}$ $(1.81 \mathrm{~g})$ and minimum weight of dry shoot (0.65g) was observed in $\mathrm{T}_{2} \mathrm{~L}_{1}$ i.e. (IBA 4000 ppm and $10 \mathrm{~cm}$ length).

However, the interaction effect of IBA concentrations, diameter and length of cutting $(\mathrm{T} \times \mathrm{D} \times \mathrm{L})$ was found to be non significant.

\section{Shoot to root ratio at 90DAP}

Data regarding shoot to root ratio of pomegranate cutting as influenced by diameter of cutting (D), length of cutting (L), IBA concentrations $(\mathrm{T})$ and their interaction are presented in Table 7.

\section{Effect of cutting diameter (D)}

Significantly highest shoot to root ratio (1.45) was recorded in $\mathrm{D}_{2}$ i.e. (1.5 cm diameter) and lowest shoot to root ratio (1.14) was observed in $\mathrm{D}_{1}$ i.e. (1 cm diameter $)$.

\section{Effect of cutting length (L)}

Among the length of cutting, highest shoot to 
root ratio (1.36) was recorded in treatment $\mathrm{L}_{3}$ i.e. (20 cm length), and lowest shoot to root ratio (1.01) was found in $\mathrm{L}_{1}$ i.e. $(10 \mathrm{~cm}$ length).

\section{IBA concentration $(T)$}

With respect to IBA concentrations, significantly highest shoot to root ratio per cutting (1.23) was recorded in treatment $\mathrm{T}_{1}$ i.e. (IBA $3000 \mathrm{ppm}$ ) and lowest (1.02) in $\mathrm{T}_{3}$ i.e. (IBA 5000 ppm).

\section{Interaction effect of $(\mathrm{L} \times \mathrm{D})$}

The interaction of length and diameter of cutting shows significant variation on shoot to root ratio. Highest shoot to root ratio (1.49) was recorded in interaction of $\mathrm{L}_{3} \mathrm{D}_{2}$ i.e. (length $20 \mathrm{~cm}$ and $1.5 \mathrm{~cm}$ diameter) and lowest shoot to root ratio (0.98) was observed in interaction of $\mathrm{L}_{1} \mathrm{D}_{1}$ i.e. (length $10 \mathrm{~cm}$ and $1 \mathrm{~cm}$ diameter). However, Interaction of IBA concentration and diameter of cutting was found to be non significant on shoot to root ratio.

\section{Interaction effect of $(\mathbf{T} \times \mathbf{L})$}

The interaction of IBA concentration and length of cutting shows significant variation on shoot to root ratio. Significantly highest shoot to root ratio (1.42) was recorded in interaction of $\mathrm{T}_{1} \mathrm{~L}_{3}$ i.e. (IBA $3000 \mathrm{ppm}$ and 20 $\mathrm{cm}$ length) and lowest shoot to root ratio (0.85) was observed in interaction of $\mathrm{T}_{3} \mathrm{~L}_{1}$ i.e. (IBA $5000 \mathrm{ppm}$ and $10 \mathrm{~cm}$ length). However, the interaction of IBA concentrations, diameter and length of cuttings $(\mathrm{T} \times \mathrm{D} \times \mathrm{L})$ do not show significant variation on shoot to root ratio per cutting. In general maximum shoot to root ratio was observed in $\mathrm{D}_{2}, \mathrm{~L}_{3}, \mathrm{~T}_{1}, \mathrm{~L}_{3} \mathrm{D}_{2}$ and $\mathrm{T}_{1} \mathrm{~L}_{3}$ due to maximum growth of shoot with available food material in early stage as compared to other treatments.

Significantly highest shoot to root ratio is due to early sprouting, root initiation and more food material is available for better growth and development of shoot and root. Similarly, IBA also induce early rooting and sprouting which helps to maximum growth of shoot and root and ultimately maximum shoot to root ratio. These results are in accordance with the findings of Hartmann et al., (2002) and Kabir (2017).

\section{Number of leaves at 30DAP}

Effect of cutting diameter (D), length of cutting (L), IBA concentrations (T) and their interactions on number of leaves per cutting are presented in Table 8 .

\section{Effect of cutting diameter (D)}

It is evident from the data that, diameter of cutting significantly affected on number of leaves per cutting. The number of leaves per cutting was highest (23.85) with treatment $\mathrm{D}_{2}$ i.e. (1.5 cm diameter) and lowest number of leaves (21.34) was recorded in $\mathrm{D}_{1}$ i.e. $(1 \mathrm{~cm}$ diameter). This might be due to vigorous rooting system of the hardwood cuttings enabled to absorb more nutrients and produce more leaves. Okunlola and Ibironke (2013).

\section{Effect of cutting length ( $L$ )}

Significantly maximum number of leaves (26.02) was recorded in $\mathrm{L}_{3}$ i.e. (20 cm length), and minimum number of leaves (20.35) was observed in $\mathrm{L}_{2}$ i.e. (15 cm length). It might be due to optimum utilization of carbohydrate, sugars and photosynthates for the growth of the plant at early stages. Similar results were also recorded by Pooja et al., (2013).

\section{IBA concentration $(T)$}

Maximum number of leaves (24.64) was recorded in treatment $T_{1}$ with application of IBA $3000 \mathrm{ppm}$ and minimum number of leaves (21.31) was recorded in $\mathrm{T}_{2}$ i.e. (IBA $3000 \mathrm{ppm})$. 
This might be due to the vigorous rooting system which might be induced by IBA enabling the cuttings to absorb more nutrients and thereby producing more leaves (Stacato $e t$ al., 2003). These findings are in close conformity with the findings of Nicoloso et al., (2001) in Pfaffia glomerata and Jadhav et al., (2003) in Patchouli.

\section{Interaction effect of $(\mathbf{L} \times \mathbf{D})$}

Significantly maximum number of leaves (29.00) was recorded in interaction of $\mathrm{L}_{3} \mathrm{D}_{2}$ i.e. (length $20 \mathrm{~cm}$ and $1.5 \mathrm{~cm}$ diameter) and minimum number of leaves (20.24) was observed in $\mathrm{L}_{2} \mathrm{D}_{2}$ i.e. (length $15 \mathrm{~cm}$ and 1.5 cm diameter).

\section{Interaction effect of $(T \times D)$}

Significantly highest number of leaves (26.17) was recorded with interaction of $\mathrm{T}_{1} \mathrm{D}_{2}$ i.e. (IBA $3000 \mathrm{ppm}$ and $1.5 \mathrm{~cm}$ diameter) which was followed by $\mathrm{T}_{3} \mathrm{D}_{2} \quad(24.11)$ and $\mathrm{T}_{1} \mathrm{D}_{1}$ (23.11) and minimum number of leaves (19.57) was observed in treatment combination of $\mathrm{T}_{3} \mathrm{D}_{1}$ i.e. (IBA $5000 \mathrm{ppm}$ and $1 \mathrm{~cm}$ diameter).

\section{Interaction effect of $(T \times L)$}

The interaction effect of length of cutting and IBA concentration on number of leaves shows significant variation. Interaction of $\mathrm{T}_{1} \mathrm{~L}_{3}$ i.e. (IBA $3000 \mathrm{ppm}$ and $20 \mathrm{~cm}$ length) was recorded maximum number of leaves (30.73) which was statistically at par with the interactions of $\mathrm{T}_{3} \mathrm{~L}_{3}$ (24.70), $\mathrm{T}_{2} \mathrm{~L}_{3}$ (22.63) and minimum number of leaves (19.23) was observed in $\mathrm{T}_{2} \mathrm{~L}_{2}$ i.e. (IBA 4000 ppm and 15 cm length)

\section{Interaction effect of $(T \times D \times L)$}

The interaction of IBA concentrations, length and diameter of cuttings show significant variation on number of leaves per cutting. The interaction of $\mathrm{T}_{1} \mathrm{D}_{2} \mathrm{~L}_{3}$ recorded significantly maximum number of leaves (36.33) which was statistically at par with the interactions of $\mathrm{T}_{3} \mathrm{D}_{2} \mathrm{~L}_{3}$ (28.66), $\mathrm{T}_{1} \mathrm{D}_{1} \mathrm{~L}_{3}(25.51)$ and minimum number of leaves (17.86) per cuttings was observed in interaction of $\mathrm{T}_{3} \mathrm{D}_{1} \mathrm{~L}_{1}$. With respect to number of leaves per cutting at 30 DAP give significant variation. Treatment $\mathrm{D}_{2}$, $\mathrm{L}_{3}, \mathrm{~T}_{1}$ and interactions of $\mathrm{L}_{3} \mathrm{D}_{2}, \mathrm{~T}_{1} \mathrm{D}_{2}, \mathrm{~T}_{1} \mathrm{~L}_{3}$, and $\mathrm{T}_{1} \mathrm{D}_{2} \mathrm{~L}_{3}$ recorded highest values.

\section{Number of leaves at 60 DAP}

The data regarding number of leaves per cutting at 60 DAP as influenced by diameter of cutting (D), length of cutting (L), IBA concentrations $(\mathrm{T})$ and their interaction are presented in Table 8. It was evident from the data that, cutting diameter was non significant effect on number of leaves per cutting.

\section{Effect of cutting length (L)}

With respect to cutting length, significantly maximum number of leaves (40.77) was recorded in treatment $\mathrm{L}_{3}$ i.e. (20 cm length) and minimum (34.12) was found in $\mathrm{L}_{1}$ i.e. $(10$ cm length).

The highest number of leaves is associated with the number of sprouts as well as length of sprout per cutting at 60 DAP, which depends in hydrolysis of reserve food materials and proper shoot and root balance. (Kaur and Kaur 2016).

\section{IBA concentration ( $T$ )}

Significantly, maximum number of leaves (39.50) was observed in $T_{1}$ application of IBA $3000 \mathrm{ppm}$ and minimum number of leaves (35.25) was recorded in $\mathrm{T}_{2}$ i.e. (IBA 4000 $\mathrm{ppm}$ ). This might be due to the vigorous rooting induced by IBA enabling the cuttings to absorb more nutrients and thereby producing more leaves, hence highest number of leaves were observed. These findings are in 
close conformity with the findings of Nicoloso et al., (2001) in Pfaffia and Jadhav et al., (2003) in Patchouli.

\section{Interaction effect of $(\mathrm{L} \times \mathrm{D})$}

With respect to interaction effect, maximum number of leaves (43.53) was recorded in interaction of $\mathrm{L}_{3} \mathrm{D}_{2}$ i.e. (length $20 \mathrm{~cm}$ and 1.5 $\mathrm{cm}$ diameter) which was statistically at par with interactions of $\mathrm{L}_{3} \mathrm{D}_{1}$ (38.02) and $\mathrm{L}_{2} \mathrm{D}_{2}$ (37.02).

However, the minimum number of leaves (33.06) was observed in interaction of $\mathrm{L}_{1} \mathrm{D}_{2}$ i.e. (length $10 \mathrm{~cm}$ and $1.5 \mathrm{~cm}$ diameter).

\section{Interaction effect of $(\mathbf{T} \times \mathbf{D})$}

Significantly, maximum number of leaves (40.35) was recorded with interaction of $\mathrm{T}_{1} \mathrm{D}_{2}$ i.e. (IBA $3000 \mathrm{ppm}$ and $1.5 \mathrm{~cm}$ diameter) which was followed by $T_{1} D_{1}$ (38.64) and $T_{3} D_{2}$ (37.68) and minimum number of leaves (34.93) was observed in treatment combination of $\mathrm{T}_{2} \mathrm{D}_{1}$ i.e. (IBA $4000 \mathrm{ppm}$ and $1.5 \mathrm{~cm}$ diameter).

\section{Interaction effect of $(T \times L)$}

The interaction effect of length of cutting and IBA concentration on number of leaves shows significant variation. Significantly highest number of leaves (30.73) was recorded in interaction of $\mathrm{T}_{1} \mathrm{~L}_{3}$ i.e. (IBA $3000 \mathrm{ppm}$ and 20 $\mathrm{cm}$ length) which was at par with the interactions of $\mathrm{T}_{3} \mathrm{~L}_{3}$ (24.70) and $\mathrm{T}_{2} \mathrm{~L}_{3}$ (22.63). However, minimum number of leaves (19.23) was observed in $\mathrm{T}_{2} \mathrm{~L}_{2}$ i.e. (IBA $4000 \mathrm{ppm}$ and $15 \mathrm{~cm}$ length).

\section{Interaction effect of $(T \times D \times L)$}

Significantly maximum number of leaves (48.33) was recorded with the interaction $\mathrm{T}_{1} \mathrm{D}_{2} \mathrm{~L}_{3}$ which was statistically at par with the interactions of $\mathrm{T}_{1} \mathrm{D}_{1} \mathrm{~L}_{2}(40.40), \mathrm{T}_{1} \mathrm{D}_{1} \mathrm{~L}_{3}$ (40.06) and minimum number (31.33) for leaves per cuttings was observed in interaction of $\mathrm{T}_{2} \mathrm{D}_{2} \mathrm{~L}_{1}$.

\section{Number of leaves at 90DAP}

Effect of cutting diameter (D), length of cutting (L), IBA concentrations (T) and their interaction on number of leaves per cutting are presented in Table 8 effect of cutting diameter on number of leaves per cutting was found to be non significant.

\section{Effect of cutting length $(\mathrm{L})$}

Significantly highest number of leaves (26.02) was recorded in treatment $\mathrm{L}_{3}$ i.e. $(20 \mathrm{~cm}$ length) and lowest number of leaves (20.35) per cutting was found in $\mathrm{L}_{2}$ i.e. (15 cm length). Significantly maximum number of leaves per cutting might be due to wood maturity of cutting which probably reserves high starch and sugar at initial stages (Pooja et al., 2013).

\section{IBA concentration ( $T$ )}

Significantly maximum number of leaves (24.64) was observed in treatment $\mathrm{T}_{1}$ i.e. application of IBA $3000 \mathrm{ppm}$ which was followed by $\mathrm{T}_{3}$ (21.84) and minimum number of leaves (21.31) was recorded in treatment $\mathrm{T}_{2}$ i.e. (IBA $4000 \mathrm{ppm}$ ). This might be due to the vigorous rooting induced by IBA enabling the cuttings to absorb more nutrients and thereby producing more leaves, hence highest number of leaves were observed. These findings are in close conformity with the findings of Nicoloso et al., (2001) in Pfaffia and Jadhav et al., (2003) in Patchouli.

\section{Interaction effect of $(L \times D)$}

According to data on number of leaves as influenced by interaction of the length and diameter of cutting was found to be 
significant. Significantly maximum number of leaves (29.00) were recorded in interaction of $\mathrm{L}_{3} \mathrm{D}_{2}$ i.e. (length $20 \mathrm{~cm}$ and $1.5 \mathrm{~cm}$ diameter), while minimum number of leaves (20.24) were observed in interaction of $\mathrm{L}_{2} \mathrm{D}_{2}$ i.e. (length $15 \mathrm{~cm}$ and $1.5 \mathrm{~cm}$ diameter).

\section{Interaction effect of $(\mathbf{T} \times \mathbf{D})$}

Significantly maximum number of leaves (26.17) were recorded with interaction of $\mathrm{T}_{1} \mathrm{D}_{2}$ i.e. (IBA $3000 \mathrm{ppm}$ and $1.5 \mathrm{~cm}$ diameter), which was closely followed by interaction of $\mathrm{T}_{3} \mathrm{D}_{2}$ (24.11) and $\mathrm{T}_{1} \mathrm{D}_{1}$ (23.11), whereas minimum number of leaves (19.57) were observed in treatment combination of $\mathrm{T}_{3} \mathrm{D}_{1}$ i.e. (IBA $5000 \mathrm{ppm}$ and $1 \mathrm{~cm}$ diameter).

\section{Interaction effect of $(T \times L)$}

The interaction effect of length of cutting and IBA concentration on number of leaves at 90 DAP shows significant variation. The interaction $\mathrm{T}_{1} \mathrm{~L}_{3}$ i.e. (IBA $3000 \mathrm{ppm}$ and 20 $\mathrm{cm}$ length) was recorded significantly maximum number of leaves (30.73) which was at par with interactions of $\mathrm{T}_{3} \mathrm{~L}_{3}$ (24.70) and $\mathrm{T}_{2} \mathrm{~L}_{3}$ (22.63) and minimum number of leaves (19.23) were observed in interaction of $\mathrm{T}_{2} \mathrm{~L}_{2}$ i.e. (IBA $4000 \mathrm{ppm}$ and $15 \mathrm{~cm}$ length).

\section{Interaction effect of $(T \times D \times L)$}

The interaction $T_{1} D_{2} L_{3}$ recorded significantly maximum number of leaves (36.33) which was statistically at par with interactions of $\mathrm{T}_{3} \mathrm{D}_{2} \mathrm{~L}_{3}$ (28.66), $\mathrm{T}_{1} \mathrm{D}_{1} \mathrm{~L}_{3}$ (25.51) and minimum number (17.86) for leaves per cuttings were observed in interaction of $\mathrm{T}_{3} \mathrm{D}_{1} \mathrm{~L}_{1}$ at $90 \mathrm{DAP}$.

\section{Leaf area $\left(\mathrm{cm}^{2}\right)$}

The data regarding leaf area per cutting at survival (90DAP) of pomegranate cuttings was influenced by diameter of cutting (D), length of cutting (L), IBA concentrations (T) and their interaction presented in Table 9.

\section{Effect of cutting diameter (D)}

Significantly highest leaf area $\left(36.86 \mathrm{~cm}^{2}\right)$ per cutting was registered with treatment $\mathrm{D}_{2}$ i.e. $(1.5 \mathrm{~cm}$ diameter) and lowest leaf area (31.57 $\left.\mathrm{cm}^{2}\right)$ was recorded in treatment $\mathrm{D}_{1}$ i.e. $(1 \mathrm{~cm}$ diameter). Increase in leaf area might be due to early sprouting and maximum leaf area. Similar result was also reported by (Taiz and Zeiger 1998) and (Kaur and Kaur 2016).

\section{Effect of cutting length ( $L)$}

Significantly highest leaf area $\left(38.36 \mathrm{~cm}^{2}\right)$ was recorded in treatment $\mathrm{L}_{3}$ i.e. (20 cm length) and lowest leaf area $\left(34.04 \mathrm{~cm}^{2}\right)$ was observed in $\mathrm{L}_{1}$. i.e. $(10 \mathrm{~cm})$.

\section{IBA concentration $(T)$}

Significantly maximum leaf area $\left(36.06 \mathrm{~cm}^{2}\right)$ was observed in treatment $\mathrm{T}_{1}$ i.e. application of IBA $3000 \mathrm{ppm}$ and minimum leaf area $\left(34.15 \mathrm{~cm}^{2}\right)$ was recorded in treatment $\mathrm{T}_{3}$ i.e. (IBA 5000 ppm). Significantly maximum leaf area is due to maximum sprouts per cutting, cutting size and IBA concentration. Similarly, the number of green leaves is the most important growth character that has direct impact on total leaf area. Since, number of green leaves was significantly influenced by diameter, length of cutting and IBA concentrations.

\section{Interaction effect of $(\mathbf{L} \times \mathbf{D})$}

Significantly maximum leaf area $\left(39.80 \mathrm{~cm}^{2}\right)$ was recorded in interaction of $\mathrm{L}_{3} \mathrm{D}_{2}$ i.e. (length $20 \mathrm{~cm}$ and $1.5 \mathrm{~cm}$ diameter) which was statistically at par with the interactions of $\mathrm{L}_{3} \mathrm{D}_{1}\left(36.93 \mathrm{~cm}^{2}\right)$ and $\mathrm{L}_{2} \mathrm{D}_{2}\left(36.75 \mathrm{~cm}^{2}\right)$ and minimum leaf area $\left(34.02 \mathrm{~cm}^{2}\right)$ was observed in interaction of $\mathrm{L}_{1} \mathrm{D}_{2}$ i.e. (length $10 \mathrm{~cm}$ and $1.5 \mathrm{~cm}$ diameter). 


\section{Interaction effect of $(T \times D)$}

Significantly maximum leaf area $\left(38.69 \mathrm{~cm}^{2}\right)$ was recorded with interaction of $\mathrm{T}_{2} \mathrm{D}_{2}$ i.e. (4000 ppm IBA and $1.5 \mathrm{~cm}$ diameter) and minimum leaf area $\left(33.40 \mathrm{~cm}^{2}\right)$ was observed in treatment combination of $\mathrm{T}_{3} \mathrm{D}_{1}$ i.e. (5000 ppm IBA and $1 \mathrm{~cm}$ diameter).

Pomegranate cuttings of $1.5 \mathrm{~cm}$ diameter and $20 \mathrm{~cm}$ length when treated with IBA 3000 ppm concentration solution for 15- 20 second gives significantly minimum days to sprout, highest sprouting percentage, maximum number of branches, maximum length and diameter of shoot, highest number of leaves per cutting, highest leaf area and finally shoot to root ratio.

\section{References}

Hartman, Kester and Davies (1990). Plant propagation; principles and practices $5^{\text {th }}$ edition Prince-Hall, London, 727.

Hartman, Kester, Davies and Geneva (2002). Plant propagation; principles and practices $6^{\text {th }}$ edition Prince-Hall International editions, Englewood Cliffs, New Jersey, USA.

Jadhav, B.J, Mahurkar, V.K. and Kale, V.S. (2003). Effect of time and severity of pruning on growth and yield of guava (Pisidium guajava L.) cv. Sardar. Orissa J. of Hort. 2003. 30:2, 83-86

Kabir M.A., Prince M.H., Karim R., Rahman S., Billah K.M.M. and Tanjina Hasnat G.N.T. (2017). Vegetative propagation of Punica granatum by stem cuttings using non-mist propagator. Int. J. Agr. Syst. 5(2): 185-197.

Kamboj S., Singh K., Singh S. and Gandhi N. (2017) Effect of Indole Butyric Acid on rooting and vegetative parameters of pomegranate (Punica granatum L.) cuttings. Int. Conf. on Recent Innovations in Sci. Agri. Eng. \&
Management ISBN: 978-93-86171-80-1.

Kaur and Kaur., (2018). Determination of suitable cutting size for rooting of Pear cuttings $c v$. Patharnakh. International Journal of Agriculture Science, Vol. 10 (12), 6445-6448.

Leaky, R.R.B., Mohammed, H.R.S., (1985) The effect of stem length on root initiation in sequential single node cuttings of Triplochitonsleroxylon K. chum. J. Horticultu. Sci., 60(5), 431-437

Levin, G.M. 2006. Pomegranate roads:a Soviet botanist's exile from Eden. pp. 15-183. B.L.Baer (ed.), Floreat Press, Forestville, CA.

Mar. M., 2000, Pomegranate plants materials. Genetics resources and breeding, a review options Mediterranean's series A. Seminnaires Mediterranean's, 42: 5562.

Okunlola and Ibironke A. (2013). The effect of cutting types and length on rooting of Durantana repens in the nursery. Global Journal of Human Social Science Vol. 13, Issue. 3.

Pooja H.M., Vasundhara M., Diwarkar Y., Sreeramu B.S., Harisha and Rajshekar. (2013). Standardization of length of cuttings for vegetative propagation of Japanese honeysuckle (Lonicera japonica). The Asian Journal of Horticulture, Vol. 8 (1), 21-23.

Shukla H.S., Tripathi V.K., Awasthi R.D. and Tripathi A.K. (2010). Effect of IBA, PHB and Boron on rooting and shoot growth of hard wood stem cuttings of peach. International Journal of Applied Agricultural Research. Volume: 5 No. 4 (2010) pp. 467-473.

Singh K.K., Choudhary T. and Kumar A. (2014). Effect of various concentrations of IBA and NAA on the rooting of stem cuttings of Mulberry (Morus Alba L.) under mist house condition in Garhwal hill region. Indian Journal of Hill Farming 27(1): 125-131. 


\section{How to cite this article:}

Basir Ahmad Ahmadi, G. M. Waghmare and Bhosale, A. M. 2021. Effect of Different Concentrations of Indole-3-butyric Acid and Different Cutting Size (Length and Diameter) on Shoot Growth of Pomegranate (Punica granatum L.) Cuttings. Int.J.Curr.Microbiol.App.Sci. 10(02): 2913-2946. doi: https://doi.org/10.20546/ijcmas.2021.1002.324 